\title{
Gold Nanoparticles as X-Ray, CT, and Multimodal Imaging Contrast Agents: Formulation, Targeting, and Methodology
}

\author{
Matthew M. Mahan and Amber L. Doiron (i) \\ Department of Biomedical Engineering, Binghamton University (SUNY), Binghamton, NY 13902, USA \\ Correspondence should be addressed to Amber L. Doiron; adoiron@binghamton.edu
}

Received 28 July 2017; Accepted 22 January 2018; Published 7 March 2018

Academic Editor: Faheem Ahmed

Copyright (c) 2018 Matthew M. Mahan and Amber L. Doiron. This is an open access article distributed under the Creative Commons Attribution License, which permits unrestricted use, distribution, and reproduction in any medium, provided the original work is properly cited.

\begin{abstract}
Computed tomography (CT) is among the most popular medical imaging modalities due to its high resolution images, fast scan time, low cost, and compatibility with all patients. CT scans of soft tissues require the localization of imaging contrast agents (CA) to create contrast, revealing anatomic information. Gold nanoparticles (AuNP) have attracted interest recently for their use as CT CA due to their high X-ray attenuation, simple surface chemistry, and biocompatibility. Targeting molecules may be attached to the particles to allow for the targeting of specific cell types and disease states. AuNP can also be readily designed to incorporate other imaging contrast agents such as rare earth metals and dyes. This review summarizes the current state-of-the-art knowledge in the field of AuNP used as X-ray and multimodal contrast agents. Primary research is analyzed through the lens of structure-propertyfunction to best explain the design of a particle for a given application. Design specification of particles includes size, shape, surface functionalization, composition, circulation time, and component synergy. Key considerations include delivery of a CA payload to the site of interest, nontoxicity of particle components, and contrast enhancement compared to the surrounding tissue. Examples from literature are included to illustrate the strategies used to address design considerations.
\end{abstract}

\section{Introduction}

Medical imaging is a critical component in the diagnosis and treatment of disease. X-ray imaging was the first modern imaging technique, but rapid advancement of technology has led to the development of other clinically employed methods including ultrasound (US) [1], magnetic resonance (MR) imaging [2], computed tomography (CT) [3], positron emission tomography (PET) [4], PET/CT [5], and PET-MRI [6]. These modalities differ in image quality metrics and application-specific factors such as depth-related image quality, resolution, signal-to-noise ratio, contrast, and sensitivity, as well as practical considerations such as safety, cost, and availability. In many clinical situations, an imaging contrast agent (CA) may be employed to enhance differentiation between two tissue types or to highlight disease-specific anatomical features or functional processes at the molecular, cellular, or tissue level. Colloidal gold is a unique material investigated as a CA in several medical imaging modalities, as summarized in Table $1[7,8]$.
Historically, gold solutions were used in stained glass windows and as a purple dye [9]. Colloidal gold was first described by Faraday in 1857 [10]. Gold nanoparticles (AuNP) are now at the forefront of developments in chemistry, biology, and medicine. Their high biocompatibility and versatile surface properties allow gold nanoparticles to be a safe platform for many biomedical technologies. Gold nanoparticles have been employed as optical biosensors [11], drug delivery vehicles [12], and imaging contrast agents [13] and laser-based treatments [14]. Their simple formulation and reactive surface allow for a variety of molecules including drugs, targeting peptides or proteins, contrast agents, or other moieties to be attached. The morphology of particles is diverse: spheres $[15,16]$, hollow shells [17], star-shapes [18], rods [19], clusters [20], and cubes [21] have all been formulated. The size of particles can range from $1 \mathrm{~nm}$ to over $100 \mathrm{~nm}$. Both size and shape have important effects on the optical properties of the particle as well as its fate in vivo.

Gold nanoparticles were first investigated as an X-ray CA by Hainfeld, who imaged organs and vasculature of mice by 
TABLE 1: Imaging modalities achieved using gold nanoparticles by inclusion of various moieties or formulation methods.

\begin{tabular}{ll}
\hline Imaging modality & Formulation method \\
\hline X-ray/CT & $\begin{array}{l}\text { Gold nanoparticles } \\
\text { Inclusion of heavy metals (gadolinium, } \\
\text { iron oxide) } \\
\text { Core-shell structure with high acoustic } \\
\text { impedance }\end{array}$ \\
Ultrasound & $\begin{array}{l}\text { Inclusion of fluorescent dyes } \\
\text { Fluorescence }\end{array}$ \\
Photoacoustic & $\begin{array}{l}\text { Inclusion of fluorescent dyes } \\
\text { Nuclear imaging }\end{array}$ \\
\hline
\end{tabular}

injecting bare AuNP intravenously $[22,23]$. The attachment of chemical moieties and targeting molecules allowed for use of these particles as multimodal contrast agents and in active targeting of disease states. A variety of capping agents or formulation methods available can increase the circulation time of AuNP, allowing for wider imaging windows. AuNP are also capable of carrying a drug or nucleic acid load [15] or mediating light-based treatments [14]. Recent reviews of therapeutic uses of AuNP are available elsewhere [24-27].

\section{Gold Nanoparticles in X-Ray Imaging}

In addition to being effective in scattering visible light, gold has a high X-ray attenuation coefficient at the energy levels utilized for clinical X-ray and CT [28]. Research-use only gold nanoparticle formulations are available commercially as Xray contrast agent AuroVist ${ }^{\mathrm{TM}}$ from nanoprobes at sizes of 1.9 and $15 \mathrm{~nm}$. Additionally, a number of innovative formulations of gold nanoparticles are under development for varied physiological applications to achieve targeted, high contrast $\mathrm{X}$-ray images for diseases diagnosis.

2.1. X-Ray/Computed Tomography. X-ray imaging was invented by Wilhelm Röntgen in 1895. It utilizes high-energy electromagnetic radiation to create images of internal structures. Today it is the most widely used method of medical imaging, accounting for $50-75 \%$ of all medical imaging done [29]. X-ray imaging is considered safe and cost-effective if the radiation dose is monitored and limited over the lifetime of the patient. An important breakthrough in 1973 resulted in the development of CT [30], which allows for 3-dimensional (3D) reconstructions of X-ray images by rotating the detector and the X-ray source about the imaged body.

When traveling through the body, X-rays undergo attenuation, the loss of beam intensity, due to photoelectric absorption or scattering. Attenuation is described by the Beer-Lambert law:

$$
I=I_{0} e^{-\mu x}
$$

where $I_{0}$ is initial beam intensity, $\mu$ is the material's characteristic absorption coefficient, and $x$ is the thickness of the tissue through which X-rays travel. The attenuation coefficient $\mu$ is described by the following equation:

$$
\mu=\frac{\rho Z^{4}}{A E^{3}},
$$

where $\rho$ is density, $Z$ is the atomic number, $A$ is the atomic mass, and $E$ is the X-ray energy. Attenuation is often measured in Hounsfield units (HU), normalized to the attenuation of water. The scale assigns an arbitrary value of 0 $\mathrm{HU}$ to water and scales linearly. Viewing this equation, it is apparent that the energy of the X-ray scan will have an effect on $\mathrm{HU}$; therefore $\mathrm{HU}$ between different experimental groups are not directly comparable.

$\mathrm{X}$-ray beams can have several different interactions with matter, but it is worth highlighting the two that predominate at clinically relevant levels of energy. Photoelectric absorption occurs when an X-ray photon interacts with an inner-shell ( $\mathrm{K}$ or L) electron of an atom. If the energy of the incident photon is greater than that of the electron, then the electron is ejected and the atom absorbs the photon completely. The inner-shell vacancy causes a cascade of electrons as outer-shell electrons fill inner-shell vacancies, releasing secondary radiation in the process. The ejected electron is absorbed by the surrounding tissue and is a main cause of biological damage in X-ray imaging. The energy threshold required to eject an inner-shell electron is referred to as the absorption edge. A spike in the mass attenuation of the bulk material is observable at these thresholds. The K-edge of several common contrast agents is within clinical CT range, including iodine $(33.2 \mathrm{keV})$, barium (34.7 keV), and gold (80.7). K-edge imaging, the detection of contrast agents using measurements of their characteristic X-ray attenuation profiles, has been used to detect AuNP in vivo [31]. Schirra utilized this property to create high contrast particles for CT scanning. $120 \mathrm{~nm}$ polysorbate cores with a lipid encapsulation were used to entrap 2-4 nm AuNP. Formulated particles provided high contrast in CT phantoms and in vivo studies [32]. This material property can be exploited to increase signal-to-noise ratio. The second type of interaction is Compton scattering. This occurs when a photon strikes a low-energy outer-shell electron, ejecting the electron and scattering the photon. Scattered Compton photons are a radiation hazard and degrade the overall image quality.

2.2. Contrast in $X$-Ray Imaging. Contrast in $\mathrm{X}$-ray imaging is derived from the difference in mass attenuation between two tissues. Materials with a high atomic number or density such as bone absorb more X-rays. Lowering the energy of the Xray beam creates a higher contrast between two tissue types because photoelectric events predominate at lower energy $(<50 \mathrm{kVp})$ [33]. Contrast agents play an important role in allowing higher energy, safer scans with high contrast by introducing high atomic number media into the body.

$\mathrm{X}$-ray contrast agents have been widely investigated over the last 20 years [34]. Iodine is the most common radiopaque element used in X-ray imaging owing to its high atomic number compared to biological tissues. For contrast media, iodine is included in compounds as triiodobenzene in either monomer or dimer structures. The agents can be 
ionic or nonionic owing to the hydrophilic residues attached to the aromatic ring. Iodinated contrast medium is in an aqueous solution with concentrations of 150 to $400 \mathrm{mg} \mathrm{I} / \mathrm{mL}$, equivalent to 0.4 to $1.0 \mathrm{~mol} / \mathrm{L}$. Its high osmolarity as well as a high viscosity can lead to poor patient tolerance. Dimeric iodine-based CA show an increase in retention in the kidneys [35] and can cause renal injury [36]. When comparing low-osmolarity CA with ones isotonic to blood plasma, a metastudy found a just-significant difference in contrast-induced nephropathy incidence [37]. The Food and Drug Administration also warns that low thyroid activity has been reported in infants who receive iodinated contrast media [38]. Iodine-based CA have also recently been linked to amplification in DNA damage during CT scans [39]. Currently, several iodinated contrast agent formulations are used clinically, including iohexol (Omnipaque ${ }^{\mathrm{TM}}$, GE Healthcare), iopromide (Ultravist ${ }^{\mathrm{TM}}$, Bayer Healthcare), ioxaglate (Hexabrix $^{\mathrm{TM}}$, Mallinckrodt Imaging), iobitridol (Xenetix ${ }^{\mathrm{TM}}$, Guerbet), and iomeprol (Imcron ${ }^{\mathrm{TM}}$, Bracco).

2.3. Cancer Targeted Gold Nanoparticles Formulations. Many researchers have used targeted gold nanoparticles to achieve contrast for the diagnostic imaging of cancer in vitro and in vivo in animal models. These approaches may employ the use of antibodies, proteins or peptides, nucleic acids, or other biomolecules. In an approach based on cancerous cells' increased metabolism and a requirement for glucose at higher rates than surrounding cells, researchers have coated gold nanoparticles with glucose analogues. Aydogan and colleagues targeted human alveolar epithelial cancer cells (A-549) in vitro using AuNP conjugated with 2-deoxy-Dglucose (2-DG). Cells were allowed to incubate with targeted AuNP before being imaged. Glucose allowed for an increased uptake of AuNP to cells, resulting in a 4-fold increase in $\mathrm{CT}$ attenuation in these targeted cells over nontargeted trials [40]. Li et al. similarly described attachment of 2-DG to AuNP and subsequent uptake into A-549 cells in vitro. Transmission electron microscopy images confirmed internalization of AuNP after incubation. A comparison between 1-deoxy-D-glucose and 2-DG showed 4-fold CT attenuation increase, and thereby higher uptake, to A-549 cells by 2DG-functionalized AuNP [41]. Furthermore, polyethylene glycol- (PEG-) functionalized AuNP coated with 2-[18F]fluoro-2-deoxy-D-glucose were used to target tumors owing to their higher metabolism compared to normal tissue. A mouse model was used to assess the targeting ability in vivo. Tumors continued to display significant contrast over nontargeted connective tissue 7 days after injection before being eliminated [42].

Many studies have successfully attached antibodies to the surface of gold nanoparticles for active targeting of an antigen on cancerous cells. Popovtzer synthesized AuNP conjugated with UM- 49 antibodies, which specifically targeted squamous cell carcinomas (SCC). An in vitro study was performed using targeted and nontargeted AuNP incubated with a variety of SCC cell types. CT attenuation was 4fold higher in trials with targeted particles and SCC cells [43]. In a separate approach, Eck et al. used two sizes, 28 and $38 \mathrm{~nm}$, of PEGylated AuNP functionalized with antimouse CD4 antibodies to target the lymph nodes of mice. AuNP were injected into the tail vein of mice and imaged at 1 and 48 hours postinjection. The targeted $38 \mathrm{~nm}$ AuNP provided a 2 -fold increase in contrast of lymph tissue, but interestingly the nontargeted $38 \mathrm{~nm}$ particles provided the second highest one. The authors suggested PEGylated particles may be taken up by macrophages and transported to the lymph nodes [44]. Conversely, Hainfeld et al. found that PEGylated AuNP conjugated with anti-Her2 antibody accumulated on the tumor periphery of Her2+ mice in drastically higher quantities than nontargeted particles, based on CT attenuation data. AuNP were injected intravenously into Her2+ and Her2- tumor-bearing mice and imaged using CT, showing a twofold increase in attenuation [45]. Reuveni et al. attached antiepidermal growth factor receptor (EGFR) antibodies to PEGylated AuNP in order to target squamous cell carcinomas. Particles were injected into the tail vein of mice bearing a xenografted tumor and compared to passively targeted AuNP. Contrast in the tumors was measured up to 6 hours later. Similar attenuation was measured for both passively and actively targeted AuNP within 0-3 hours, but within 3-6 hours active targeting had a twofold higher contrast [46]. Kao et al. functionalized PEGylated AuNP with cetuximab, an EGFR-targeting drug to target and image human lung cancer line A-549. Cetuximab is a chimeric monoclonal antibody that inhibits EFGR. A tumor was xenografted into a mouse model and the particles were injected intravenously. CT imaging showed the majority of particles accumulated in the liver and bladder, although the tumor was visible up to 4 hours postinjection [47].

In addition to using antibodies, proteins or peptides can be attached to gold nanoparticles to target cancerous cells. Chanda et al. attached bombesin peptide, which targets gastrin-releasing peptide receptors that are overexpressed in several cancers, to AuNP. Human prostate tumors were xenografted into mice and the AuNP injected intraperitoneally to assess their ability to target a tumor in vivo. CT attenuation was found to be 4 -fold higher within the tumor up to 6 hours after injection of particles [48]. Additionally, Angiopep-2, another tumor-targeting peptide, was used by Hao et al. Poly(lactic glycolic acid) (PLGA) nanoparticles coated with Angiopep-2-functionalized AuNP were injected into a mouse and compared against untargeted particles. Formulated particles showed a linear attenuation curve with a slope of $0.1 \mathrm{mg} \mathrm{Au} \mathrm{ml}^{-1} \mathrm{HU}^{-1}$. Tumors showed higher attenuation for both particles, although the control group had some signal the authors attributed to the enhanced permeability and retention effect (EPR) [49].

In a separate approach, Tian and colleagues conjugated arginine-glycine-aspartic acid (RGD) peptides to hollow gold nanospheres. The widely used RGD peptides target $\alpha_{5} \beta_{3}$ integrin receptors overexpressed by tumor blood vessels. The targeting ability of RGD-AuNP was assessed by injecting the particles into rabbits with a xenografted VX2 liver tumor and imaging after 60 minutes. Particles were injected via several routes and in conjunction with different iodized oils to improve liver accumulation; the highest levels were found with intra-arterial injection and lipiodol. A twofold 
CT attenuation increase was observed in the tumor tissue under these conditions [50]. RGD was also employed by Zhu et al., who used generation 5 (G5) poly(amidoamine) (PAMAM) dendrimers as a scaffold to entrap AuNP and RGD peptide. This polymer can be used as a scaffold on which to reduce AuNP as well as PEG using 1-ethyl-3-(3dimethylaminopropyl)carbodiimide (EDC) chemistry. The so-called generation of the PAMAM dendrimers refers to its size; for example, generation 2 dendrimers have a lower size and molecular weight than generation 5 . The particles were assessed for their CT contrast and targeting ability in vitro. CT phantoms showed an attenuation similar to Omnipaque. Trials using free RGD and U87MG cells overexpressing $\alpha_{5} \beta_{3}$ integrin receptors showed that blocking these receptors prevented attachment of the particles, so targeting was concluded to be specific [51].

Aptamers represent a newer approach to active targeting. Aptamers are typically synthetic, short strands of oligonucleic acids or oligopeptides (15-60 amino acids) that bind to a specific target molecule. Kim et al. attached a prostate-specific membrane antigen ribonucleic acid-aptamers to AuNP with the goal of targeting prostate cancer cells. Targeting efficiency of the AuNP was assessed in vitro after incubation with prostate-specific membrane antigen-overexpressing cells. CT attenuation was threefold higher than that of the control cells [52]. AS1411 aptamers attached to AuNP were used in one study to investigate their tumor-targeting ability. These particles had a linear attenuation curve with a slope of $0.027 \mathrm{mM} \mathrm{Au} \mathrm{HU}{ }^{-1}$. CL1-5 tumor-bearing mice were used to assess this targeting ability in vivo. A high CT attenuation at the tumor site indicated high accumulation of particles at the tumor site [53]. Additionally, Melancon et al. compared the targeting ability of EGFR-targeting aptamers to targeting antibodies on hollow AuNP. Squamous cell carcinomas were created by injection of cells into mice and particles injected intravenously. Higher CT attenuation was observed for aptamer particles at 4 and 24 hours postinjection, showing much promise for this aptamer-based approach to active targeting [54].

Receptor-antigen binding is another biological mechanism that can be taken advantage of for targeting of nanoparticles for imaging purposes. Folic acid receptor, a glycosylphosphatidylinositol anchored cell surface receptor, is overexpressed on the majority of cancer tissues, compared to healthy tissues and organs. Folic acid- (FA-) conjugated G5 PAMAM dendrimers were used as a template to attach AuNP in order to target human lung adenocarcinoma cells (SPC-A1). The particles were used both in vitro and in vivo in a xenografted tumor mouse model. TEM images of in vitro incubations showed internalized AuNP. Tumors continued to display significant X-ray attenuation up to 6 hours postinjection, with up to a fourfold increase when an intratumoral injection route was used [55]. Liu and colleagues used a similar approach in creating silver-gold alloys with amine-terminated generation 5 (G5.NH2) dendrimers as stabilizers. The dendrimers were functionalized with FA for targeting of cancer cells. Human epithelial carcinoma cell lines (KB cells) over- and underexpressing FA receptors were incubated with the particles in vitro. However, CT attenuation of $\mathrm{KB}$ cells exposed to targeted particles was not significantly different over nontargeted particles in this study [56]. In another dendrimer-based approach, Cai et al. used poly $(\gamma$-glutamic acid) (PGA) dendrimers attached to $\mathrm{Fe}_{3} \mathrm{O}_{4}$ nanoparticles to entrap AuNP. These particles were further functionalized with $\mathrm{FA}$ to target $\mathrm{KB}$ cells. In vivo studies showed targeted particles created a twofold increase in CT attenuation after 24 hours [57]. Similarly, Chen et al. entrapped AuNP in a G5.NH2 dendrimer functionalized with FA to target $\mathrm{KB}$ cells. Entrapped particles showed a linear attenuation curve with a slope of $0.2 \mathrm{mM} \mathrm{Au} \mathrm{Hu}^{-1}$. In vitro and in vivo studies were conducted to test the targeting abilities. Contrast was 2-fold higher over control 24 hours postinjection [58]. A follow-up study replaced FA with the RGD peptide. After demonstrating significant cellular uptake in vitro, particles were assessed in a mouse tumor. Included were trials where mice were injected with free RGD to block $\alpha_{5} \beta_{3}$ receptors. The CT attenuation of tumor tissue was 1.5fold higher than control between 1 and 20 hours postinjection [59]. Zhou created gold nanoclusters with silica oxide shells conjugated with FA for targeting of MGC803 (gastric cancer) cells in a xenografted mouse model. Despite the shell, a linear attenuation curve with a slope of $0.74 \mathrm{mM} \mathrm{Au} \mathrm{HU}^{-1}$ was observed. Particles injected by the tail vein showed high contrast 6 hours postinjection at the tumor site, although attenuation was not quantified [60].

Various other biomolecule-based approaches to targeting exist in the literature as well. Sun et al. investigated the tumor-targeting ability of glycolic chitosan- (GC-) capped AuNP [61] based on previous literature using GC-targeting of tumors $[62,63]$. In vitro studies demonstrated that the formulated particles had similar CT attenuation to eXIA 160, a commercial CT contrast agent. An in vivo study using mice compared heparin-coated AuNP, a nontargeted vehicle, to GC-AuNP. GC-AuNP were found to have a 2-fold CT signal increase over control 2 hours postinjection [61]. Furthermore, gold-coated $\mathrm{Fe}_{2} \mathrm{O}_{3}$ particles were conjugated with lectin, a glycan-targeting molecule, and used to image colorectal cancer in a mouse model. Several lectins were used, including Ricinus communis agglutinin I (RCA), concanavalin A (ConA), and wheat germ agglutinin (WGA). RCA and WGA exhibited similar targeting capability as measured by CT imaging 24 hours postinjection [64]. One study used G5.NH2 dendrimers modified with lactobionic acid (LA), which specifically binds asialoglycoprotein (ASGP), as a scaffold to reduce AuNP. Hepatocellular carcinoma (HCC) was used to test targeting ability owing to the cell line's overexpression of ASGP receptors. This targeting ability was assessed in vivo in a mouse model. LA-conjugated scaffolds showed a 1.5-fold higher tumor CT attenuation after 2 hours when injected intravenously [65]. Zeng et al. functionalized AuNP with LA to target HCC cell line HepG2. This targeting ability was assessed by comparing internalization versus an ASGPcell line (HeLa). Only HepG2 showed internalization after incubation in vivo. X-ray images of a phantom were obtained that showed a steep linear attenuation slope of $0.00016 \mathrm{mM}$ Au $\mathrm{HU}^{-1}$ [66].

$\mathrm{Li}$ et al. created $\mathrm{Fe}_{3} \mathrm{O}_{4}$ core gold nanostars with a polyethyleneimine layer to entrap hyaluronic acid (HA). 
HA was used to target CD44 receptors overexpressed by cancer cells. This targeting was tested both in vitro using CD44+ and CD44- cells and in vivo in a mouse model. In vitro trials showed 1.5 -fold increase in $\mathrm{CT}$ attenuation over control at concentrations above $2.50 \mathrm{mM} \mathrm{Au}$. In vivo CT scans conducted showed significant contrast at the tumor site 10 minutes postinjection [18]. Bisphosphonate-conjugated AuNP were formulated to target microcalcifications in breast tissue and improve cancer detection in vivo using CT imaging. Bisphosphonate specifically binds hydroxyapatite, the mineral component of calcifications. Mammary tumorbearing mice were used as a model and particles were injected into the mammary tissue. Particles allowed imaging of previously undetectable microcalcification and provided CT contrast for up to 48 hours [67]. One study by Meir has even demonstrated that cancerous tissue may be targeted without the use of small molecules. AuNP were incubated with T cells transformed to express a melanoma-specific T-cell receptor before being injected intravenously into mice bearing human melanoma xenografts. The internalized particles were tracked using CT and cells were found to accumulate in the melanomas, while nontransformed cells did not [68].

Cancer remains an important focus of health science study in general and AuNP research in particular. Many of these studies opted to localize particles by functionalizing them with cancer cell-specific molecules such as antibodies and antigens. While this has been shown to be effective at localizing particles within the tumor bulk, many of these studies are conducted in mice. It remains to be seen whether such targeting methods are viable in human subjects.

2.4. Thrombus Targeted Gold Nanoparticles. Kim et al. investigated the thrombus localization of GC-coated AuNP. Clots were induced in mice before particles were injected. CT imaging was able to visualize plaques 30 minutes after induction of the thrombus and 5 minutes after injection of particles and also 48 hours and 3 weeks later without further injection of AuNP. Application of tissue plasminogen activator, the clinical preference for clot breakdown, led to a decrease in CT signal at the clot areas [69]. A follow-up study added fibrintargeting peptides to the formulation. Fib-GC-AuNP had superior targeting to GC-AuNP in vitro. Cerebral embolisms were induced by injecting fluorescently labeled thrombi into the cerebral artery of mice. Fluorescence imaging was used to colocalize visualization of the particles and found a significant signal at the site of clots [70]. Ghann et al. coated AuNP with Lisinopril, an angiotensin-converting enzyme (ACE) inhibitor, and targeted $\mathrm{ACE}$ in vivo to track the progression of heart disease. Particles were injected into mice and targeted to the lungs and heart. Thioctic acid attached to Lisinopril was required to stabilize the particles. Particles provided significant contrast and pretreatment with free Lisinopril resulted in low signal scans, confirming direct targeting [71].

2.5. Macrophage Targeted Formulations. Macrophages are important markers of diseases such as heart disease and cancer. Cormode et al. created high-density lipopolysaccharide capsules with AuNP cores to assess macrophage burden and plaque stability. Atherosclerosis was induced in mice, and the particles were injected 24 hours before sacrifice. Spectral CT scanning of the aorta showed high CT attenuation at areas known to accumulate plaque such as the arch and the bifurcation [72]. From the same group, van Schooneveld et al. formulated a gold core/silica shell with surface lipid molecules and PEG. In vitro phagocytic studies and in vivo liver visualization studies were conducted. Fluorescence microscopy images of macrophages revealed particle internalization and images taken 24 hours after intravenous injection to mice revealed an increase in CT signal in the liver [73]. De Wilde's group investigated using CT to monitor the stability of arterial plaques. They observed AuNP were phagocytosed by macrophages. Exploiting this, they tracked the macrophages and quantified their accumulation within the plaques [16]. Allijn et al. incorporated AuNP into low-density lipopolysaccharide capsules and tracked their fate in vivo. These capsules displayed a linear attenuation curve with a slope of $0.25 \mathrm{mM} \mathrm{Au} \mathrm{HU}^{-1}$. Mice bearing B16F10 tumors were injected with LDL-AuNP and imaged using CT. A significant signal was measured at the tumor site and the liver. Fluorescence activated cell sorting revealed that the majority of particles were taken up by macrophages [74]. Qin et al. used G5.NH2 dendrimers functionalized with fluorescein isothiocyanate as scaffolds to reduce AuNP. Macrophage uptake was assessed in vivo using Apolipoprotein-E knockout mice. Atherosclerotic vessels had significantly higher CT signal 6 hours postinjection. Hematoxylin and eosin staining of resected arteries confirmed macrophage uptake of the particles [75].

AuNP coated in heparin were tested for their ability to accumulate in Kupffer cells. These particles were used as contrast agents to image a mouse liver in vivo. In a comparison with eXIA160, formulated particles had a higher CT signal intensity within the liver [76]. Zhao et al. reduced AuNP onto $\mathrm{Fe}_{3} \mathrm{O}_{4}$ particles using mercaptosuccinic acid. The synthesized particles were intended to accumulate in Kupffer cells and were investigated in vivo for their diagnostic potential of three stages of liver diseases: fatty liver, cirrhotic liver, and HCC. In the case of HCC, particles acted as a negative contrast by providing contrast to the liver parenchyma but not the tumor location [77].

Furthermore, Chhour et al. used 11-mercaptoundecanoic acid (11-MUDA) as a stabilizer for formulated AuNP in order to track macrophages. These particles were used to label monocytes ex vivo before injection into atherosclerotic mice. Over the course of 5 days, CT signal at the aorta increased significantly pointing to an accumulation of AuNP and therefore the macrophages [78].

Many of these macrophage targeting methods rely on encapsulation or cellular uptake. Strategies such as the use of lipopolysaccharides have great potential for targeting by exploiting the physiological mechanisms. These encapsulated particles still display attenuation curves comparable to other CT contrast agents. It remains to be seen whether these targeting strategies provide enough contrast in human subjects.

2.6. Bone Targeted Formulations. Zhang sought to quantify microdamage to bone using AuNP as CA in CT imaging. By 
functionalizing AuNP with glutamic acid, particles were able to be targeted towards bone by chelating calcium ions at the surface of bone crystals. Bovine bone resections were used as a model, and particle accumulation was visualized using scanning electron microscopy. Authors note the potential of the particle to be used as an X-ray contrast agent [79]. Another study encapsulated $\mathrm{Fe}_{3} \mathrm{O}_{4}$ and AuNP within a silica matrix before incorporation into a calcium phosphate-based bone replacement cement. This formulation was tested in vitro for its physical properties and in vivo for its incorporation into a mouse femoral condyle defect. The cement was able to be visualized up to 8 weeks after implantation [80]. While bone itself does not require a $\mathrm{CA}$, these methods of targeting may allow the imaging of bone defects or tissue implants.

2.7. Kidney Targeted Formulations. Renal imaging is an important factor in the diagnosis of kidney disease and cancer. Wang et al. created small $(<2 \mathrm{~nm})$ AuNP clusters coated with bovine serum albumin (BSA) in order to diagnose kidney disease. The formulated particles were compared in vitro to iopromide as well as being used for CT imaging in vivo. Similar CT attenuation was achieved with lower concentrations of the particles compared to iopromide. A high resolution image of the kidneys was possible using CT scanning 2 hours postinjection [81].

\subsection{Blood Pool Imaging Gold Nanoparticle Formulations.} AuNP have been utilized as blood pool contrast agents in lieu of localization for a particular disease. Kattumuri et al. utilized gum arabic as a capping agent for AuNP. CT attenuation was measured at 80 and $140 \mathrm{kVp}$ using phantoms; the particles were also injected in a swine model to assess biodistribution. Particles at both CT energies had a linear relationship to CT attenuation and accumulated in the liver, lungs, and spleen [82]. A follow-up study by the same group used the gum arabic-coated particles to image swine organs. CT scans of the liver and spleen revealed an increase in $\mathrm{HU}$ after intravenous particle injection [83]. In a different approach, Cai et al. investigated PEGylated $38 \mathrm{~nm}$ AuNP as blood pool contrast by injecting the particles into Balb/c mice. Significant CT contrast was measured in the right ventricle, inferior vena cava, liver, spleen, and kidneys at all time points between 0.1 and 24 hours after injection. Histological staining of these organs confirmed the presence of AuNP [84]. AuNP have been shown to be superior to iodine-based CA for imaging of vasculature. PEGylated AuNP injected into mice were compared to iodine injections at a tube voltage of $60 \mathrm{kVp}$. AuNP persisted in the vasculature for up to 24 hours and had a higher contrast than did iodine, which was eliminated after 6 hours [85].

Polymers are also capable of long circulation times that allow for vascular imaging. Peng et al. reported use of G5.NH2 dendrimers modified with diatrizoic acid (DA) to entrap AuNP. DA is an iodine containing compound designed to enhance CT contrast. Particles were injected intravenously into mice to evaluate their CT contrast. Particles containing DA showed 2-fold over scaffolds without DA and 10-fold higher contrast over Omnipaque [86]. In another case, PEGylated generation 4 PAMAM dendrimers with reduced AuNP were formulated to be used as a blood pool contrast agent. Mice were injected with formulated particles or iopamidol in a comparison of contrasting ability and halflife. HU values were similar for both CA, although iopamidol accumulated within the bladder due to renal elimination [87].

Hayashi et al. formulated gold clusters with a silica shell to visualize lymph nodes and lymphatic vessels using CT scanning. The CT attenuation of the particles was first compared to Iopamiron before injection into mice. Formulated particles displayed greater CT attenuation than Iopamiron and lymphatic vessels in phantoms and were used to visualize nodes 18 hours postinjection [88].

Although nontargeted formulations do not have a method of tissue localization, they still require long enough circulation times to be of use in a clinical setting. Particles used in a blood pool setting may be relied upon for perfusion studies, while imaging of organs relies upon natural physiological processes to localize particles.

\subsection{Passively Targeted Gold Nanoparticle Formulations.} While not targeted in the active sense, AuNP can be formulated such that their accumulation in certain tissues is predictable. A small particle with a long circulation time in vivo can lead to the accumulation of particles in tumors owing to their leaky vasculature. This EPR effect allows researchers to accumulate particles within tumors without actively targeting them. Hainfeld et al. injected AuNP into a mouse model with a brain tumor xenograft to assess uptake. A high concentration ( $4 \mathrm{~g} \mathrm{Au} / \mathrm{kg}$ ) was used to achieve a high tumor uptake ( $1.5 \% \mathrm{w} / \mathrm{w} \mathrm{Au})$. Tumors continued to be visible on CT scans up to 8 days after intravenous injection [89].

Another popular strategy is the coating of AuNP with PEG, which changes their route of clearance from the renal system to the reticuloendothelial system (RES). PEGylation increases circulation time of the particles within the body and can allow particles to be passively targeted to tumor cells. Clearance by the RES also causes particle accumulation in the liver and spleen. Maltzahn et al. compared PEGylated AuNR with a commercial iodine-based CT CA Isovue-370 (iopamidol). AuNR were injected both intratumorally and intravenously into a tumor-bearing mouse. The formulated particles had a nonlinear CT attenuation higher than that of iopamidol, and intravenously injected particles had a 17-hour half-life [90]. Ashton et al. demonstrated tumor accumulation of PEGylated AuNP in an interesting study using dual-energy CT. AuNP were injected and allowed to accumulate in tumors for 2 days before the injection of iodine nanoparticles. The iodine particles acted as blood pool CA, while the AuNP provided contrast within the tumors [91]. PEGylated hollow AuNP are also capable of targeting tumors passively as shown by Park et al. After injection into the tail vein, particle location was assessed using CT imaging and inductively coupled plasma (ICP) mass spectroscopy. Although particles could be detected within the body, authors were unable to image the tumor owing to a low concentration of particles [17]. Naha et al. used PEGylated gold-silver alloy particles to passively target breast cancer tumors and perform dual-energy mammography CT scans. Mice were injected 
with the particles via the tail vein and imaged at 30,60, and 120 minutes postinjection. Contrast was significantly higher in the blood and tumor at all time points. The gold-silver alloy reduced the amount of silver ion leaching over silver nanoparticles alone [92].

Wang targeted tumors using a G5.NH2 PAMAM dendrimer scaffold with reduced gold particles. The dendrimers were then acetylated to neutralize the positive surface potential. Targeting was assessed in vivo using a mouse xenograft tumor. Particles were injected intratumorally and intraperitoneally; both routes showed a high CT attenuation in the tumor indicating targeting by the EPR effect [93]. Li used G5.NH2 PAMAM dendrimers with gadolinium chelator tetraazacyclododecane tetraacetic acid (DOTA-NHS) as scaffolds to attach gadolinium, AuNP, and PEG. The particles were assessed for their ability to accumulate in tumors using CT imaging. CT attenuation was highest in the tumor 100 minutes postinjection, although significance was not shown. The particles also had a much higher accumulation in the liver, lungs, and spleen [94]. Another study utilizing G5.NH2 dendrimers modified with AuNP and PEG imaged the blood pool of mice using CT as well as a xenograft tumor targeted via the EPR effect. The particles showed higher CT attenuation than Omnipaque in phantoms and displayed 1.5fold contrast over control up to 6 hours after injection [95]. Generation 2 PAMAM dendrimers have also been utilized by Liu in a similar study using the scaffold as a platform to attach AuNP and PEG. These low generation dendrimers were injected both intravenously and interperitoneally to assess their tumor accumulation and body clearance. The particles were found to accumulate in tumor and the bladder, although much higher CT attenuation was observed in the latter. In a comparison to Omnipaque, formulated particles remained in the bladder for longer time periods [96].

Polymers are a popular tool to allow the attachment of several functionalizing molecules. Zhou et al. used PEGylated branched polyethylenimine as a scaffold to synthesize AuNP. Subcutaneous injection into healthy rats allowed the particles to be used as blood pool CA, while intravenous injection into a tumor model showed accumulation at the cancer site owing to the EPR effect [97]. AuNP loaded into 1,2-distearoyl-sn-glycero-3-phosphoethanolamine- $N$-[methoxy(polyethylene glycol)-2000] (DPSEPEG2000) micelles were used to target tumors in vivo. Particles were injected intravenously into CT26 tumorbearing mice and imaged using CT. The highest CT signal was observable at the liver, although the tumor site also exhibited high signal [98]. Wang et al. encapsulated $\mathrm{Fe}_{3} \mathrm{O}_{4}$ nanoparticles in dipalmitoylphosphatidylcholine (DPPC)/ cholesterol/DSPE-PEG 2000 liposomes coated in AuNP and PEG. These particles were injected intravenously to assess their accumulation in tumors. Particles did enhance CT contrast, although the majority of particles accumulated in the organs [99]. Li reported a gold nanocluster assembly encapsulated by polyacrylic acid (PAA)/calcium phosphate. Particles were injected intravenously into mice to assess their fate in vivo. Tumor tissue had the highest CT signal and gold concentration as assessed by ICP atomic emission spectroscopy [20].
PEG has been shown to play a major role in determining the location and accumulation of nanoparticles in vivo, and a wide variety of PEGylated particles have been investigated for tumor accumulation. Fullerenes have been used as a template to reduce AuNP and were functionalized using PEG attached via a $\mathrm{pH}$-sensitive hydrazine bond. This allowed particles to be targeted to tumors owing to the EPR effect, but upon reaching the tumor site PEG would be cleaved allowing uptake directly into cells. This process was evaluated in vivo using a tumor-bearing mouse. CT signal was observable 8 hours postinjection. Histological staining of resected tumor cells revealed internalization of particles [100]. Hayashi designed large $(<100 \mathrm{~nm})$ gold core cluster/silica shell particles with surface PEG for passive targeting of tumors in an animal model. Particles were injected intravenously, and CT imaging was performed at 3 and 24 hours. Tumor tissue had a 2.5 -fold difference in attenuation 24 hours postinjection. Histological staining of tumor tissue confirmed the presence of formulated particles [88]. Ke coated perfluorooctylbromide capsules with gold to create a dual ultrasound/CT CA agent (discussed below). Particles were also PEGylated to enhance accumulation in a mouse tumor in vivo. CT imaging after intravenous administration of the particles showed highest accumulation in the liver and kidneys [101].

Various other surface molecules and capping agents are capable of increasing circulation time enough to allow tumor accumulation of particles. Chanda et al. performed a green synthesis of AuNP using cinnamon phytochemicals as reducing agents. TEM images showed internalization to cancer cell lines PC-3 and MCF-7 after in vitro incubation. CT imaging phantoms showed a linear attenuation curve of $54.1 \mu \mathrm{M} \mathrm{HU}^{-1}$. They concluded the cinnamon-capped particles could circulate for long enough to accumulate in the lungs [102]. Luo et al. attached Cyanine5.5 (a nearinfrared fluorescent dye) and a black hole quencher to a GCcapped AuNP and assessed its ability to target tumors in vivo. Particles were injected intravenously into a tumor-bearing mouse and assessed after 24 hours. Tumors were targeted by the EPR effect and showed a fivefold CT attenuation increase over control [103]. Prussian blue-coated AuNP were used in a study assessing their use as CT/photoacoustic CA. Particles were injected into a tumor-bearing mouse and CT imaging was performed over a 24-hour period. Despite the lack of surface functionalization, CT signal continued to increase over the time course. Biodistribution studies revealed the highest accumulation was in the spleen [104].

Sousa et al. designed human serum albumin-coated AuNP as a potential treatment for prion disease. The particles were investigated for their ability to cross the blood brain barrier by intravenous injection into mice. Ex vivo CT scans of the brain showed the highest CT signal was located at the thalamus and hypothalamus [105].

Clark et al. undertook a study to establish a CT imaging protocol to quantify angiogenesis and tumor blood volume. AuroVist AuNP were injected into mice 4 days before liposomal iodine. Scans were conducted using microCT daily up to 6 days postinjection. The difference in CT attenuation between iodine and gold as well as the uptake of particles due to the EPR allowed tumor vasculature to be imaged. 
AuNP initially created an attenuation of up to $800 \mathrm{HU}$ in the vasculature but decreased to $10 \mathrm{HU}$ in the tumor tissue. After injection of the iodine, an attenuation of $25 \mathrm{HU}$ was observed in the vasculature, which allowed for the differentiation of vasculature from tumor tissue [106].

As with nontargeting, these passive targeting methods rely on both the EPR effect or the RES system to localize particles within the organs of interest. The liver and spleen, which are responsible for dealing with matter picked up by the RES, thus may be naturally targeted by AuNP. Given long enough circulation time, the EPR effect can also be relied upon to localize particles to tumors.

\section{Multimodal Particles}

Gold particles can be designed to be used with multiple imaging modalities. A given modality may not be appropriate for all tissue types; for example, $\mathrm{X}$-ray imaging is generally not appropriate for soft tissue due to poor X-ray attenuation. The ability to utilize multiple imaging modalities with one contrast agent allows clinicians to gather varied information with one contrast agent and increase clinical acceptance. Particles can be made multimodal by changing the design to include molecules with the desired properties. Potential modalities include magnetic resonance (MR) imaging, fluorescence imaging, ultrasound, photoacoustic imaging, and nuclear imaging.

3.1. Gold Nanoparticle-Based Contrast Agents for Magnetic Resonance Imaging. Discussion of the mechanisms of MRI are beyond the scope of this paper, but an excellent summary can be found in MRI basic principles and applications [107]. Gadolinium-based contrast agents (GBCA) are the current standard for MRI contrast agents owing to their high paramagnetism. There are 9 GBCA that are clinically approved; however, there are multiple reports of toxicity [108-110]. Superparamagnetic iron oxide $\left(\mathrm{Fe}_{3} \mathrm{O}_{4}\right)$ nanoparticles (SPIOs) have been investigated as liver-specific contrast agents [111, 112]. There are reports of increases in reactive oxygen species caused by SPIOs [113], so additional modifications to formulations may be required for complete biocompatibility.

Several formulation methods exist for including paramagnetic components. Two compounds may be combined together to create a heterogenous structure or in a core-shell fashion. One study used gold nanorods, rather than spherical particles, coated with a polypyrrole shell and seeded with reduced $\mathrm{Fe}_{3} \mathrm{O}_{4}$ crystals. The particles were tested in vitro for their contrasting properties; both $\mathrm{T}_{1}$ and $\mathrm{T}_{2}$ relaxation was investigated as well as CT contrast. $\mathrm{T}_{2}$ relaxivity was much higher than $\mathrm{T}_{1}\left(128.57 \mathrm{mM}^{-1} \mathrm{~s}^{-1}\right.$ and $7.99 \mathrm{mM}^{-1} \mathrm{~s}^{-1}$, resp.) pointing to effectiveness as a $\mathrm{T}_{2}$ contrast agent. $\mathrm{CT}$ values varied linearly with concentration. Authors note that further in vivo studies to assess imaging efficacy and biodistribution are underway [114]. An $\mathrm{Fe}_{3} \mathrm{O}_{4}$ core/Au nanostar shell coated with polyethyleneimine (PEI) was designed in a study by Li et al. Testing was performed in vitro as well as in vivo in tumorbearing mice. The particles had a high CT attenuation and were used as a $\mathrm{T}_{2}$ contrast agent in vivo although scans were only compared after 10 minutes [18]. Amendola et al. created
$\mathrm{Au} / \mathrm{Fe}_{3} \mathrm{O}_{4}$ alloy nanoparticles by laser ablation of a gold/iron bulk material. These particles were PEGylated and assessed in imaging phantom for their CT and MR contrast ability. The particles were also investigated for toxicity towards cells and their biodistribution in mice [115]. Kim et al. coated a AuNP/ $\mathrm{Fe}_{3} \mathrm{O}_{4}$ heteroparticle with the amphiphilic polymer poly(DMA- $r$-mPEGMA- $r$-MA), which contains a PEG moiety. An animal model bearing a liver tumor was selected to ascertain whether the tumor could be distinguished from the liver parenchyma. CT images were unclear, but authors noted a 1.5-fold signal enhancement detectable 24 hours postinjection. MR contrast was larger than that of commercial CA Resovist ${ }^{\circledR}$ at similar concentrations [116]. The aforementioned study by Zhao et al. first synthesized $\mathrm{Fe}_{3} \mathrm{O}_{4}$ particles by coprecipitation, capped with mercaptosuccinic acid (DMSA) before AuNP were reduced onto their surface. Both MR and CT contrast in the liver parenchyma was at a peak 30 minutes postinjection and was still enhanced after 6 hours, although significance was not assessed [77].

Entrapment has been utilized as a particle creation strategy for MR/CT particles. One such method first formulated thiolated $\mathrm{Fe}_{3} \mathrm{O}_{4}$ /silica composites by self-assembly of amphiphilic block PAA copolymers and $\mathrm{Fe}_{3} \mathrm{O}_{4}$ cross-linked by 3-mercaptopropyltrimethoxysilane. These were then used as scaffolds to reduce AuNP and used to image mouse tumors in vivo. $\mathrm{CT}$ and $\mathrm{MR}$ images taken at 5 and 30 minutes postinjection did show an increase in contrast, although significance was not determined [117]. Cai et al. used an $\mathrm{Fe}_{3} \mathrm{O}_{4}$ core with a dendrimer trilayer (PGA/poly(L-lysine) (PLL)/PGA) to entrap gold particles. These AuNP were utilized in seed-mediated growth, and the effect of $\mathrm{Au} / \mathrm{Fe}_{3} \mathrm{O}_{4}$ molar ratio on $\mathrm{MR}$ and $\mathrm{CT}$ signal was assessed. At a ratio of $2: 1$ they achieved a $\mathrm{T}_{2}$ relaxivity of $92.67 \mathrm{~s}^{-1} \mathrm{mM}^{-1}$. Their particle was assessed in vivo in a mouse model and showed a 1.5-fold increase in CT attenuation over control. A biodistribution study showed significant accumulation in the liver and spleen [57]. One study used PEGylated PEI as a scaffold to reduce AuNP. These polymers were then attached to an $\mathrm{Fe}_{3} \mathrm{O}_{4}$ particle and assessed for their contrast ability in a mouse model. Significant MR contrast was measured up to 4 hours, while CT attenuation was measured to be 1.5 -fold higher than baseline after 20 minutes [118]. Sun et al. created an $\mathrm{Au} / \mathrm{Fe}_{3} \mathrm{O}_{4}$ heteroparticle by entrapment in a PEG/polycaprolactone micelle. They injected it intravenously into mouse models with human glioblastoma multiforme tumors implanted in the flank or brain and measured passive accumulation of their particle by CT and MR scans. The heteroparticle possessed a 3 : 1 gold : iron oxide ratio by mass while still possessing a high $\mathrm{T}_{2}$ relaxivity $\left(221.92 \mathrm{~s}^{-1} \mathrm{mM}^{-1}\right)$. This gave it excellent contrast in MR scans of both areas of interest, but the CT scans showed negligible differences [119]. Wang et al. designed a large PEG micelle with encapsulated $\mathrm{Fe}_{3} \mathrm{O}_{4}$ particles and a gold shell. Formulated particles possessed a low attenuation slope of 6.7 $\mu \mathrm{M} \mathrm{HU}^{-1}$. These were injected intravenously into the tail vein of tumor-bearing mice to test the particles' contrast properties. Relatively high concentrations of the particle were required to create appropriate contrast levels in vivo, and a high accumulation of particles in the liver, lungs, and spleen was found [99]. Tian et al. studied attachment of gold 
nanoparticles to a gadolinium-organometallic framework, rather than a gold particle with gadolinium chelates. PAA acted as the active site to entrap gold particles. The composite particle displayed better MR contrast than clinically available Magnevist at lower concentrations with a similar relaxivity and similar CT attenuation to Omnipaque [120]. G5 PAMAM dendrimers can also be used to entrap other metals for the creation of multimodal particles. Wen et al. created such dendrimers with reduced gold clusters, gadolinium ions, and PEG. The particles were injected into the tail vein of mice and imaged at time points up to 45 minutes postinjection. CT and MR contrast saw a 1.5-fold increase in the liver and kidneys and a 2-fold increase in the bladder [121]. Chen created PEGylated G5 PAMAM dendrimers with reduced $4 \mathrm{~nm}$ gold clusters and gadolinium ions. The particles were injected subcutaneously into tumor-bearing mice and used as contrast agents in CT and MR imaging up to 24 hours postinjection. Contrast in both modalities continued to increase up to 24 hours, showing up to 1.5 -fold increase in CT attenuation and MRI signal [58].

Encapsulation within biocompatible materials can also be utilized to create multimodal particles. Graphene is one possible encapsulator. One group used an aerosol encapsulation process to create large $(>100 \mathrm{~nm})$ graphene particles encapsulating AuNP and $\mathrm{Fe}_{3} \mathrm{O}_{4}$ particles. The particles were assessed for their material property, including porosity, pore volume, and ability to encapsulate effectively versus associating with metallic particles. CT phantom imaging revealed an attenuation curve consistent with the mass fraction of free AuNP. MR imaging showed a linear relationship between concentration and $\mathrm{T}_{2}$ signal [122]. Another study reduced $\mathrm{Fe}_{3} \mathrm{O}_{4} \mathrm{NP}$ and AuNP onto graphene oxide (GO) sheets as well as PEG. In vivo imaging was performed by injecting particles into the tumor of mice. Imaging performed over a 5-minute period postinjection shows an increase in contrast [123]. Wu et al. encapsulated gadolinium-gold clusters in silica to create $80 \mathrm{~nm}$ nanoparticles. After injection into a mouse tumor, the particles were used as a contrast agent in MR and CT imaging. CT attenuation was increased 9-fold over preinjection values using $200 \mu \mathrm{L}$ of $40 \mathrm{mM}$ particle solution [124]. Zhang's group designed a complex particle with a large gold particle coated by a silica shell with embedded manganese oxide and gold particles. Their idea was to design a particle with components that would amplify the properties each harbors. The $\mathrm{T}_{1}$ relaxivity of the particle $\left(1.5 \mathrm{mM}^{-1} \mathrm{~s}^{-1}\right)$ was lower than commercial gadolinium-based CA (i.e., Magnevist: $4.6 \mathrm{mM}^{-1} \mathrm{~s}^{-1}$ ), but it did display 1.5-fold signal over control in vivo [125]. Liu et al. encapsulated gold nanostars in silica to allow attachment of gadolinium ions. The particles were evaluated for their CT and MR contrasting ability using phantoms. Both modalities showed a linear relationship between contrast and particle concentration [126].

Gold itself has been shown to be a viable platform for creation of a multimodal particle. Coughlin used $120 \mathrm{~nm}$ silica particles with a gold shell as platforms to attach gadolinium and PEG. These particles were injected intratumorally into mice. High contrast was observable in the tumor, although it was not quantified [127]. Zeng et al. used lipid-coated $\mathrm{AuNP}$ as a platform to attach gadolinium. Imaging phantoms were used to evaluate the particles properties as contrast agents. The $\mathrm{T}_{1}$ relaxivity of the particle was higher than that of commercially available dimeglumine gadopentetate [66]. Alric attached gadolinium particles to 1.9 and $31 \mathrm{~nm}$ gold nanoparticles. These particles were injected into mice and imaged at 10 and 30 minutes. CT imaging showed the highest gold accumulation in the renal system, while the highest MR signal was observed in the bladder [128].

Many innovative methods of combining magnetic materials with AuNP have been utilized, including heteroparticles, entrapment, encapsulation, and reduction of one onto the other. These methods have varying effects on both imaging metrics and biocompatibility and thus must be investigated further for their viability in human subjects. The marriage of these materials represents the possible synergy of the two most used clinical imaging methods and thus a step forward in their capability in diagnosis.

\subsection{Gold Nanoparticle-Based Contrast Agents for Ultrasound.} Ultrasound is an imaging modality utilizing soundwaves above the human range of hearing, usually taken as above 20 kiloHertz $(\mathrm{kHz})$. Current state of the art in ultrasound contrast agents lies in the use of microbubbles. Their use comes from a desire to image blood flow and perfusion of organ tissues and has expanded into treatment methods [129]. Schutt et al. provide an excellent review of microbubble design as well as signal and mechanical properties [130].

Researchers designing CT/US contrast agents utilize the characteristics of microbubbles combined with gold particles. Ke et al. designed a perfluorooctyl bromide- (PFOB-) gold core-shell complex. PFOB has a much higher acoustic impedance than air, enabling it to be used as US CA. The particles were used to successfully image the kidney and liver of mice [101]. Jin created a microcapsule of poly(lactic acid) containing gold and graphene oxide nanoparticles [131]. Interestingly, the amount of GO increased contrast in pulse inversion harmonic mode which creates contrast by nonlinear scattering. This suggests that the GO itself was resonating with ultrasound pulses. These capsules can also deliver a payload, as with the study by Arifin et al. that utilized alginate microcapsules to protect pancreatic islet cells in mice. The capsule allowed the pancreatic cells to maintain normal glucose levels for 6-7 weeks when injected into diabetic mice. Particles were tracked using CT and US [132]. The previously mentioned study by Zhang used a silica shell rather than a carbon-based one but it was still able to achieve an acceptable signal due to the nonlinear scattering caused by the embedded gold and manganese oxide particles [125]. Teraphongphom et al. encapsulated AuNP within a PLA microbubble. Although the payload was effectively loaded, a significant decrease in US signal at 50.0 wt\% AuNPmicrobubble was observed [8]. The encapsulation method utilized by many of these methods can have a detrimental effect on CT signal. Further work is required to maximize both CT and US contrast simultaneously.

3.3. Fluorescence. Emitted light from fluorescence can originate from a variety of sources including fluorescent dyes, quantum dots, or gold nanoparticles. It is not a commonly 
utilized clinical modality but is real-time and noninvasive. Drawbacks include photobleaching, tissue autofluorescence increasing noise, and the low penetration depth of light. However, fluorescence is widely used in research and has some clinical applications. Fluorescence can also be useful when combined with another, high resolution modality to grant colocalization information.

Gold particles may be functionalized with a dye to confer well-studied optical properties. Mesoporous silicacoated gold nanorods were loaded with indocyanine green (ICG) to increase their fluorescence signal when used in vivo. The particles were loaded into tumor-bearing mice and used as fluorescence and CT CA. Fluorescence imaging revealed a strong signal 12 hours postinjection owing to the gradual release of ICG from the mesoporous silica [103]. Zhang loaded PEG micelles with AuNP and bis(4- $(N-(2-$ naphthyl)phenylamino)phenyl)-fumaronitrile (NPAPF), an aggregation induced emission dye. The fluorescence of this dye is able to overcome the quenching effect of the AuNP when in a high water fraction liquid. These properties made it an appropriate candidate for in vivo imaging. The fluorescent signal was still highly detectable in a mouse model after 24 hours [98].

Aggregated or clustered AuNP have been shown to enhance luminescence signal over single particles alone. The above study by Li also utilized fluorescence imaging to track particles in vivo. The aggregated nature of the gold particles enhanced fluorescence signal compared to discrete nanoclusters, resulting in a 5-fold higher fluorescence intensity. The particles also created significant contrast in vivo after injection into tumor-bearing mice [20]. The above multimodal particle designed by $\mathrm{Wu}$ et al. also utilized gold clusters. This allowed the particles to be used as fluorescence contrast agents in vivo in a tumor-bearing mouse. Interestingly, these aggregated particles underwent a blue-shift in fluorescence peak compared to discrete AuNP, from 612 to $595 \mathrm{~nm}$ [124]. Zhou et al. encapsulated gold clusters in silica for fluorescence imaging in mice. Particles were injected into three separate sites on the back of tumor-bearing mice. Fluorescence imaging revealed a strong signal 6 hours postinjection, while CT imaging showed a 3-fold increase in CT attenuation [60]. Hayashi designed gold aggregates encapsulated with silica to enhance the overall fluorescent property of the particles. Authors noted the strong fluorescence enhancement of particles caused by their surface plasmon resonance. Particles still created a strong fluorescence signal 18 hours after injection into a mouse model [133]. Although fluorescence imaging is not common clinically, its simple image capture technique may point to its use in surgical settings.

3.4. Photoacoustic Imaging. Photoacoustic imaging is a relatively new method that takes advantage of the same optical properties as fluorescence imaging. Tissues are irradiated by visible or near-infrared light resulting in adiabatic expansion. This creates pressure waves, which are in turn measured and used to reconstruct an image. The modality depends upon the optical and thermal properties of the tissues. Contrast agents are utilized in cases where depth of penetration is low or there is a lack of natural contrast between tissues [134]. Chanda et al. investigated the use of cinnamon phytochemicals as an AuNP capping agent to enhance particle uptake to cancer cells. Particles were added to cells in vitro and irradiated using a tunable laser. Untreated cells showed a linear photoacoustic response, while treated cells displayed a time variant signal, indicating particle uptake and particle contrast ability [102]. The fluorescent dye Prussian blue has also been utilized as a coating to enhance photoacoustic signal. Particles were tested in agar gels as well as mice using a $765 \mathrm{~nm}$ laser. In the agar gel, increasingly thick layers of tissue were placed in the laser path to investigate signal penetration depth. High resolution was achieved in the absence of tissue, while an increasing loss of clarity was evident up to $4.3 \mathrm{~cm}$ [104]. Cheheltani et al. encapsulated AuNP into polydi(carboxylatophenoxy)phosphazene (PCPP) nanospheres. Formulated particles exhibited high CT contrast, while the absorbable wavelengths could be tuned by changing the size or amount of included AuNP [7]. Photoacoustic imaging is still a new modality, and further work is required to fully understand the parameters required for AuNP as a photoacoustic contrast agent.

3.5. Nuclear Imaging: Single Photon Emission Computed Tomography and Positron Emission Tomography. Nuclear imaging comprises two separate 3D modalities, single photon emission computed tomography (SPECT) and positron emission tomography (PET), which both operate on the same principles while utilizing different contrast agents. Both rely on patient internalization of a radiotracer comprised of an isotope and a biological targeting element. Images arise through the detection of gamma rays. The targeting component of the radiotracer gives biologically relevant information such as the metabolism of a compound. Thus while most modalities originate their signal externally, nuclear imaging signal arises internally and depends entirely on the radiotracers used.

The same isotopes traditionally utilized as nuclear imaging radiotracers can be added to AuNP to achieve multimodal imaging. One study used PEGylated, hollow gold nanospheres as platforms to attach ${ }^{64} \mathrm{Cu}$ to achieve PET imaging. Particles were injected into tumor-bearing mice either intra-arterially or intravenously along with iodinated oil to enhance tumor uptake. PET/CT scans 1 hour after injection show high contrast in the tumor area [50]. Li et al. undertook a study using both ${ }^{111} \mathrm{In}$ - or ${ }^{64} \mathrm{Cu}$-labeled AuNP for SPECT and PET imaging, respectively. The particles were loaded into $\mathrm{T}$ cells using electroporation; these cells were subsequently injected into mice for imaging. ${ }^{111}$ In-labeled cells were injected directly into the lung of mice and successfully imaged using PET/CT. ${ }^{64} \mathrm{Cu}$-labeled cells were injected into the tail vein and tracked using SPECT/CT imaging over the course of 18 hours [135]. Targeted hollow gold nanospheres carrying ${ }^{111}$ Indium were used to image tumors on the tongue of mice. Particles were targeted using EGFRtargeting aptamers and injected intravenously. SPECT/CT imaging showed a much higher signal intensity using targeted particles, even after 24 hours [54]. Although this combination 
of imaging techniques is relatively unexplored, the targeting ability and long circulation time of gold nanoparticles may offer a platform for further nuclear imaging studies.

\section{Conclusion}

Gold nanoparticles have great potential as contrast agents in a variety of imaging modalities. On their own, their high $\mathrm{X}$-ray attenuation and nontoxicity may be combined with surface molecules to enhance tissue-targeting and improve circulation time. AuNP size and reactivity also allow their accumulation in certain organ systems and tissues, especially cancerous tumors. When combined with other materials such as rare earth metals or fluorescent dyes, their use expands to include imaging modalities such as magnetic resonance imaging or ultrasound. These additional materials may add or subtract from image contrast and may create toxicity or circulation concerns. A diverse array of formulation methods including entrapment and encapsulation have been developed to overcome these design hurdles. This multimodal possibility represents a step forward in clinical diagnosis. Long circulating, nontoxic contrast agents that can reveal anatomical and disease information across multiple imaging types could ease clinical imaging burdens and simplify scanning procedures.

AuNP for use as X-ray contrast agents are currently available commercially, although they are for research use only. In order to gain clinical acceptance, further in vivo human research must be conducted. Questions still exist as to their biodistribution, circulation times, and targeting ability. Still, AuNP remain an excellent platform for X-ray contrast agents. Improvements in their ability to effectively circulate and localize at a desired area of interest will allow for their use in clinical settings.

\section{Conflicts of Interest}

The authors declare that they have no conflicts of interest.

\section{References}

[1] T. L. Szabo, Diagnostic Ultrasound Imaging: Inside Out, Academic Press, Cambridge, Mass, USA, 2004.

[2] D. W. McRobbie, MRI from Picture to Proton, Cambridge University Press, Cambridge, UK, 2007.

[3] J. Hsieh, Computed Tomography: Principles, Design, Artifacts, and Recent Advances, SPIE Press, 3rd edition, 2015.

[4] R. Nutt, "The history of positron emission tomography," Molecular Imaging and Biology, vol. 4, no. 1, pp. 11-26, 2002.

[5] M. Nahrendorf, H. Zhang, S. Hembrador et al., "Nanoparticle PET-CT imaging of macrophages in inflammatory atherosclerosis," Circulation, vol. 117, no. 3, pp. 379-387, 2008.

[6] P. S. Chandra, N. Salamon, J. Huang et al., "FDG-PET/MRI coregistration and diffusion-tensor imaging distinguish epileptogenic tubers and cortex in patients with tuberous sclerosis complex: a preliminary report," Epilepsia, vol. 47, no. 9, pp. 15431549, 2006.
[7] R. Cheheltani, R. M. Ezzibdeh, P. Chhour et al., "Tunable, biodegradable gold nanoparticles as contrast agents for computed tomography and photoacoustic imaging," Biomaterials, vol. 102, pp. 87-97, 2016.

[8] N. Teraphongphom, P. Chhour, J. R. Eisenbrey et al., "Nanoparticle loaded polymeric microbubbles as contrast agents for multimodal imaging," Langmuir, vol. 31, no. 43, pp. 11858-11867, 2015.

[9] L. B. Hunt, "The true story of Purple of Cassius - The birth of gold-based glass and enamel colours," Gold Bulletin, vol. 9, no. 4, pp. 134-139, 1976.

[10] M. Faraday, "The Bakerian lecture: experimental relations of gold (and other metals) to light," Philosophical Transactions of the Royal Society A: Mathematical, Physical \& Engineering Sciences, vol. 147, pp. 145-181, 1857.

[11] L. Nie, F. Liu, P. Ma, and X. Xiao, "Applications of gold nanoparticles in optical biosensors," Journal of Biomedical Nanotechnology, vol. 10, no. 10, pp. 2700-2721, 2014.

[12] H. Daraee, A. Eatemadi, E. Abbasi, S. F. Aval, M. Kouhi, and A. Akbarzadeh, "Application of gold nanoparticles in biomedical and drug delivery," Artificial Cells, Nanomedicine and Biotechnology, vol. 44, no. 1, pp. 410-422, 2016.

[13] L. E. Cole, R. D. Ross, J. M. Tilley, T. Vargo-Gogola, and R. K. Roeder, "Gold nanoparticles as contrast agents in X-ray imaging and computed tomography," Nanomedicine, vol. 10, no. 2, pp. 321-341, 2015.

[14] S. Hwang, J. Nam, S. Jung, J. Song, H. Doh, and S. Kim, “Gold nanoparticle-mediated photothermal therapy: current status and future perspective," Nanomedicine, vol. 9, no. 13, pp. 20032022,2014

[15] A. Ahangari, S. Mojtaba, and F. Saghatchi, "Gentamicin-gold nanoparticles conjugate: a contrast agent for X-ray imaging of infectious foci due to staphylococcus aureus," IET Nanobiotechnology, vol. 10, no. 4, pp. 190-194, 2016.

[16] D. De Wilde, B. Trachet, C. Van der Donckt et al., "Vulnerable plaque detection and quantification with gold particleenhanced computed tomography in atherosclerotic mouse models," Molecular Imaging, vol. 14, no. 6, 2015.

[17] J. Park, J. Park, E. J. Ju et al., "Multifunctional hollow gold nanoparticles designed for triple combination therapy and CT imaging," Journal of Controlled Release, vol. 207, pp. 77-85, 2015.

[18] J. Li, Y. Hu, J. Yang et al., "Hyaluronic acid-modified $\mathrm{Fe}_{3} \mathrm{O}_{4}$ at $\mathrm{Au}$ core/shell nanostars for multimodal imaging and photothermal therapy of tumors," Biomaterials, vol. 38, pp. 10-21, 2015.

[19] P. Jackson, S. Periasamy, V. Bansal, and M. Geso, "Evaluation of the effects of gold nanoparticle shape and size on contrast enhancement in radiological imaging," Australasian Physical \& Engineering Sciences in Medicine, vol. 34, no. 2, pp. 243-249, 2011.

[20] L. Li, L. Zhang, T. Wang et al., "Facile and scalable synthesis of novel spherical Au nanocluster assemblies@polyacrylic acid/calcium phosphate nanoparticles for dual-modal imagingguided cancer chemotherapy," Small, vol. 11, no. 26, pp. 31623173, 2015.

[21] Q.-Y. Lin, Z. Li, K. A. Brown et al., "Strong coupling between plasmonic gap modes and photonic lattice modes in DNAassembled gold nanocube arrays," Nano Letters, vol. 15, no. 7, pp. 4699-4703, 2015.

[22] J. F. Hainfeld, D. N. Slatkin, and H. M. Smilowitz, "The use of gold nanoparticles to enhance radiotherapy in mice," Physics in Medicine and Biology, vol. 49, no. 18, pp. N309-N315, 2004. 
[23] J. F. Hainfeld, D. N. Slatkin, T. M. Focella, and H. M. Smilowitz, "Gold nanoparticles: a new X-ray contrast agent," British Journal of Radiology, vol. 79, no. 939, pp. 248-253, 2006.

[24] L. Vigderman and E. R. Zubarev, “Therapeutic platforms based on gold nanoparticles and their covalent conjugates with drug molecules," Advanced Drug Delivery Reviews, vol. 65, no. 5, pp. 663-676, 2013.

[25] J. P. Almeida, E. R. Figueroa, and R. A. Drezek, "Gold nanoparticle mediated cancer immunotherapy," Nanomedicine: Nanotechnology, Biology and Medicine, vol. 10, no. 3, pp. 503514, 2014.

[26] C. Zhao and Z. Liu, "Application of gold nanoparticles in cancer therapy," Zhongguo Yi Xue Ke Xue Yuan Xue Bao, vol. 36, no. 3, pp. 324-329, 2014.

[27] Y. Ding, Z. Jiang, K. Saha et al., "Gold nanoparticles for nucleic acid delivery," Molecular Therapy, vol. 22, no. 6, pp. 1075-1083, 2014.

[28] C. Suplee, "X-ray mass attenuation coefficients," NIST, 2009, https://www.nist.gov/pml/x-ray-mass-attenuation-coefficients.

[29] S. Yu and A. D. Watson, "Metal-based X-ray contrast media," Chemical Reviews, vol. 99, no. 9, pp. 2353-2378, 1999.

[30] R. E. Alexander and R. B. Gunderman, "EMI and the first CT scanner," Journal of the American College of Radiology, vol. 7, no. 10, pp. 778-781, 2010.

[31] S. Si-Mohamed, D. P. Cormode, D. Bar-Ness et al., "Evaluation of spectral photon counting computed tomography K-edge imaging for determination of gold nanoparticle biodistribution in vivo," Nanoscale, vol. 9, no. 46, pp. 18246-18257, 2017.

[32] C. O. Schirra, A. Senpan, E. Roessl et al., "Second generation gold nanobeacons for robust K-edge imaging with multi-energy CT,' Journal of Materials Chemistry, vol. 22, no. 43, pp. 2307123077, 2012.

[33] R. R. Carlton and A. M. Adler, Principles of Radiographic Imaging: An Art and A Science, Delmar/Cengage Learning, Clifton Park, NY, USA, 2013.

[34] H. Katayama, K. Yamaguchi, T. Kozuka, T. Takashima, P. Seez, and K. Matsuura, "Adverse reactions to ionic and nonionic contrast media. A report from the Japanese Committee on the Safety of Contrast Media," Radiology, vol. 175, no. 3, pp. 621-628, 1990.

[35] G. Jost, H. Pietsch, P. Lengsfeld, J. Hütter, and M. A. Sieber, “The impact of the viscosity and osmolality of iodine contrast agents on renal elimination," Investigative Radiology, vol. 45, no. 5, pp. 255-261, 2010.

[36] D. C. Lenhard, H. Pietsch, M. A. Sieber et al., "The osmolality of nonionic, iodinated contrast agents as an important factor for renal safety," Investigative Radiology, vol. 47, no. 9, pp. 503-510, 2012.

[37] J. Eng, R. F. Wilson, R. M. Subramaniam et al., "Comparative effect of contrast media type on the incidence of contrastinduced nephropathy a systematic review and meta-analysis," Annals of Internal Medicine, vol. 164, no. 6, pp. 417-424, 2016.

[38] Research C for DE and Drug Safety and Availability, "FDA Drug Safety Communication: FDA advises of rare cases of underactive thyroid in infants given iodine-containing contrast agents for medical imaging," 2017, http://www.fda.gov/Drugs/ DrugSafety/ucm472782.htm.

[39] E. I. Piechowiak, J.-F. W. Peter, B. Kleb, K. J. Klose, and J. T. Heverhagen, "Intravenous iodinated contrast agents amplify DNA radiation damage at CT," Radiology, vol. 275, no. 3, pp. 692-697, 2015.
[40] B. Aydogan, J. Li, T. Rajh et al., "AuNP-DG: Deoxyglucoselabeled gold nanoparticles as X-ray computed tomography contrast agents for cancer imaging," Molecular Imaging and Biology, vol. 12, no. 5, pp. 463-467, 2010.

[41] J. Li, A. Chaudhary, S. J. Chmura et al., "A novel functional CT contrast agent for molecular imaging of cancer," Physics in Medicine and Biology, vol. 55, no. 15, pp. 4389-4397, 2010.

[42] G. Feng, B. Kong, J. Xing, and J. Chen, "Enhancing multimodality functional and molecular imaging using glucose-coated gold nanoparticles," Clinical Radiology, vol. 69, no. 11, pp. 1105-1111, 2014.

[43] R. Popovtzer, A. Agrawal, N. A. Kotov et al., "Targeted gold nanoparticles enable molecular CT imaging of cancer," Nano Letters, vol. 8, no. 12, pp. 4593-4596, 2008.

[44] W. Eck, A. I. Nicholson, H. Zentgraf, W. Semmler, and S. Bartling, "Anti-CD4-targeted gold nanoparticles induce specific contrast enhancement of peripheral lymph nodes in X-ray computed tomography of live mice," Nano Letters, vol. 10, no. 7, pp. 2318-2322, 2010.

[45] J. F. Hainfeld, M. J. O'Connor, F. A. Dilmanian, D. N. Slatkin, D. J. Adams, and H. M. Smilowitz, "Micro-CT enables microlocalisation and quantification of Her2-targeted gold nanoparticles within tumour regions," British Journal of Radiology, vol. 84, no. 1002, pp. 526-533, 2011.

[46] T. Reuveni, M. Motiei, Z. Romman, A. Popovtzer, and R. Popovtzer, "Targeted gold nanoparticles enable molecular CT imaging of cancer: an in vivo study," International Journal of Nanomedicine, vol. 6, pp. 2859-2864, 2011.

[47] H.-W. Kao, Y.-Y. Lin, C.-C. Chen et al., "Evaluation of EGFRtargeted radioimmuno-gold-nanoparticles as a theranostic agent in a tumor animal model," Bioorganic \& Medicinal Chemistry Letters, vol. 23, no. 11, pp. 3180-3185, 2013.

[48] N. Chanda, V. Kattumuri, R. Shukla et al., "Bombesin functionalized gold nanoparticles show in vitro and in vivo cancer receptor specificity," Proceedings of the National Acadamy of Sciences of the United States of America, vol. 107, no. 19, pp. 8760$8765,2010$.

[49] Y. Hao, B. Zhang, C. Zheng et al., "The tumor-targeting core-shell structured DTX-loaded PLGA@Au nanoparticles for chemo-photothermal therapy and X-ray imaging," Journal of Controlled Release, vol. 220, pp. 545-555, 2015.

[50] M. Tian, W. Lu, R. Zhang et al., "Tumor uptake of hollow gold nanospheres after intravenous and intra-arterial injection: PET/CT study in a rabbit VX2 liver cancer model," Molecular Imaging and Biology, vol. 15, no. 5, pp. 614-624, 2013.

[51] J. Zhu, F. Fu, Z. Xiong, M. Shen, and X. Shi, "Dendrimerentrapped gold nanoparticles modified with RGD peptide and alpha-tocopheryl succinate enable targeted theranostics of cancer cells," Colloids and Surfaces B: Biointerfaces, vol. 133, pp. 36-42, 2015.

[52] D. Kim, Y. Y. Jeong, and S. Jon, "A drug-loaded aptamer-gold nanoparticle bioconjugate for combined CT imaging and therapy of prostate cancer," ACS Nano, vol. 4, no. 7, pp. 3689-3696, 2010.

[53] C. Li, T. Kuo, H. Su et al., "Fluorescence-guided probes of aptamer-targeted gold nanoparticles with computed tomography imaging accesses for in vivo tumor resection," Scientific Reports, vol. 5, no. 1, 2015.

[54] M. P. Melancon, M. Zhou, R. Zhang et al., "Selective uptake and imaging of aptamer- and antibody-conjugated hollow nanospheres targeted to epidermal growth factor receptors 
overexpressed in head and neck cancer," ACS Nano, vol. 8, no. 5, pp. 4530-4538, 2014.

[55] H. Wang, L. Zheng, C. Peng, M. Shen, X. Shi, and G. Zhang, "Folic acid-modified dendrimer-entrapped gold nanoparticles as nanoprobes for targeted CT imaging of human lung adencarcinoma," Biomaterials, vol. 34, no. 2, pp. 470-480, 2013.

[56] H. Liu, M. Shen, J. Zhao et al., "Facile formation of folic acidmodified dendrimer-stabilized gold-silver alloy nanoparticles for potential cellular computed tomography imaging applications," Analyst, vol. 138, no. 7, pp. 1979-1987, 2013.

[57] H. Cai, K. Li, J. Li et al., "Dendrimer-Assisted Formation of $\mathrm{Fe}_{3} \mathrm{O}_{4} / \mathrm{Au}$ Nanocomposite Particles for Targeted Dual Mode CT/MR Imaging of Tumors," Small, vol. 11, no. 35, pp. 45844593, 2015.

[58] Q. Chen, K. Li, S. Wen et al., “Targeted CT/MR dual mode imaging of tumors using multifunctional dendrimer-entrapped gold nanoparticles," Biomaterials, vol. 34, no. 21, pp. 5200-5209, 2013.

[59] Q. Chen, H. Wang, H. Liu et al., "Multifunctional dendrimerentrapped gold nanoparticles modified with RGD peptide for targeted computed tomography/magnetic resonance dualmodal imaging of tumors," Analytical Chemistry, vol. 87, no. 7, pp. 3949-3956, 2015.

[60] Z. Zhou, C. Zhang, Q. Qian et al., "Folic acid-conjugated silica capped gold nanoclusters for targeted fluorescence/X-ray computed tomography imaging," Journal of Nanobiotechnology, vol. 11, article 17, 2013.

[61] I.-C. Sun, J. H. Na, S. Y. Jeong et al., "Biocompatible glycol chitosan-coated gold nanoparticles for tumor-targeting CT imaging," Pharmaceutical Research, vol. 31, no. 6, pp. 1418-1425, 2014.

[62] J. H. Na, S.-Y. Lee, S. Lee et al., "Effect of the stability and deformability of self-assembled glycol chitosan nanoparticles on tumor-targeting efficiency," Journal of Controlled Release, vol. 163, no. 1, pp. 2-9, 2012.

[63] Y. Zhang and M. Zhang, "Calcium phosphate/chitosan composite scaffolds for controlled in vitro antibiotic drug release," Journal of Biomedical Materials Research Part B: Applied Biomaterials, vol. 62, no. 3, pp. 378-386, 2002.

[64] X. He, F. Liu, L. Liu, T. Duan, H. Zhang, and Z. Wang, "Lectinconjugated $\mathrm{Fe}_{2} \mathrm{O}_{3} @$ Au core@ shell nanoparticles as dual mode contrast agents for in vivo detection of tumor," Molecular Pharmaceutics, vol. 11, no. 3, pp. 738-745, 2014.

[65] H. Liu, H. Wang, Y. Xu et al., "Lactobionic acid-modified dendrimer-entrapped gold nanoparticles for targeted computed tomography imaging of human hepatocellular carcinoma," ACS Applied Materials \& Interfaces, vol. 6, no. 9, pp. 6944-6953, 2014.

[66] Y. Zeng, D. Zhang, M. Wu et al., "Lipid-AuNPs@PDA nanohybrid for MRI/CT imaging and photothermal therapy of hepatocellular carcinoma," ACS Applied Materials \& Interfaces, vol. 6, no. 16, pp. 14266-14277, 2014.

[67] L. E. Cole, T. Vargo-Gogola, and R. K. Roeder, "Contrastenhanced X-ray detection of microcalcifications in radiographically dense mammary tissue using targeted gold nanoparticles," ACS Nano, vol. 9, no. 9, pp. 8923-8932, 2015.

[68] R. Meir, K. Shamalov, and O. Betzer, "Nanomedicine for cancer immunotherapy: tracking cancer-specific T-cells in vivo with gold nanoparticles and CT imaging," ACS Nano, vol. 9, no. 6, pp. 6363-6372, 2015.

[69] D.-E. Kim, J.-Y. Kim, I.-C. Sun et al., "Hyperacute direct thrombus imaging using computed tomography and gold nanoparticles," Annals of Neurology, vol. 73, no. 5, pp. 617-625, 2013.

[70] J.-Y. Kim, J. H. Ryu, D. Schellingerhout et al., "Direct imaging of cerebral thromboemboli using computed tomography and fibrin-targeted gold nanoparticles," Theranostics, vol. 5, no. 10, pp. 1098-1114, 2015.

[71] W. E. Ghann, O. Aras, T. Fleiter, and M.-C. Daniel, "Syntheses and characterization of lisinopril-coated gold nanoparticles as highly stable targeted CT contrast agents in cardiovascular diseases," Langmuir, vol. 28, no. 28, pp. 10398-10408, 2012.

[72] D. P. Cormode, E. Roessl, A. Thran et al., "Atherosclerotic plaque composition: Analysis with multicolor CT and targeted gold nanoparticles," Radiology, vol. 256, no. 3, pp. 774-782, 2010.

[73] M. M. Van Schooneveld, D. P. Cormode, R. Koole et al., "A fluorescent, paramagnetic and PEGylated gold/silica nanoparticle for MRI, CT and fluorescence imaging," Contrast Media \& Molecular Imaging, vol. 5, no. 4, pp. 231-236, 2010.

[74] I. E. Allijn, W. Leong, J. Tang et al., "Gold nanocrystal labeling allows low-density lipoprotein imaging from the subcellular to macroscopic level," ACS Nano, vol. 7, no. 11, pp. 9761-9770, 2013.

[75] J. Qin, C. Peng, B. Zhao et al., "Noninvasive detection of macrophages in atherosclerotic lesions by computed tomography enhanced with PEGylated gold nanoparticles," International Journal of Nanomedicine, vol. 9, no. 1, pp. 5575-5590, 2014.

[76] I.-C. Sun, D.-K. Eun, J. H. Na et al., "Heparin-coated gold nanopartieles for liver-specific CT imaging," Chemistry, vol. 15, no. 48, pp. 13276-13347, 2009.

[77] H. Y. Zhao, S. Liu, J. He et al., "Synthesis and application of strawberry-like $\mathrm{Fe}_{3} \mathrm{O}_{4}$-Au nanoparticles as CT-MR dualmodality contrast agents in accurate detection of the progressive liver disease," Biomaterials, vol. 51, pp. 194-207, 2015.

[78] P. Chhour, P. C. Naha, S. M. O’Neill et al., "Labeling monocytes with gold nanoparticles to track their recruitment in atherosclerosis with computed tomography," Biomaterials, vol. 87, pp. 93103, 2016.

[79] Z. Zhang, R. D. Ross, and R. K. Roeder, "Preparation of functionalized gold nanoparticles as a targeted X-ray contrast agent for damaged bone tissue," Nanoscale, vol. 2, no. 4, pp. 582586, 2010.

[80] M. Ventura, Y. Sun, V. Rusu et al., "Dual contrast agent for computed tomography and magnetic resonance hard tissue imaging," Tissue Engineering - Part C: Methods, vol. 19, no. 6, pp. 405-416, 2013.

[81] Y. Wang, C. Xu, J. Zhai et al., "Label-free Au cluster used for in vivo $2 \mathrm{D}$ and $3 \mathrm{D}$ computed tomography of murine kidneys," Analytical Chemistry, vol. 87, no. 1, pp. 343-345, 2015.

[82] V. Kattumuri, K. Katti, and S. Bhaskaran, "Gum arabic as a phytochemical construct for the stabilization of gold nanoparticles: in vivo pharmacokinetics and X-ray-contrast-imaging studies," Small, vol. 3, no. 2, pp. 333-341, 2007.

[83] E. Boote, G. Fent, V. Kattumuri et al., "Gold nanoparticle contrast in a phantom and juvenile swine. Models for molecular imaging of human organs using X-ray computed tomography," Academic Radiology, vol. 17, no. 4, pp. 410-417, 2010.

[84] Q.-Y. Cai, S. H. Kim, K. S. Choi et al., "Colloidal gold nanoparticles as a blood-pool contrast agent for X-ray computed tomography in mice," Investigative Radiology, vol. 42, no. 12, pp. 797-806, 2007.

[85] J. T. Au, G. Craig, V. Longo et al., "Gold nanoparticles provide bright long-lasting vascular contrast for CT imaging," American Journal of Roentgenology, vol. 200, no. 6, pp. 1347-1351, 2013. 
[86] C. Peng, K. Li, X. Cao et al., "Facile formation of dendrimerstabilized gold nanoparticles modified with diatrizoic acid for enhanced computed tomography imaging applications," Nanoscale, vol. 4, no. 21, pp. 6768-6778, 2012.

[87] C. Kojima, Y. Umeda, M. Ogawa, A. Harada, Y. Magata, and K. Kono, "X-ray computed tomography contrast agents prepared by seeded growth of gold nanoparticles in PEGylated dendrimer.," Nanotechnology, vol. 21, no. 24, p. 245104, 2010.

[88] K. Hayashi, M. Nakamura, and K. Ishimura, "Near-Infrared Fluorescent Silica-Coated Gold Nanoparticle Clusters for XRay Computed Tomography/Optical Dual Modal Imaging of the Lymphatic System," Advanced Healthcare Materials, vol. 2, no. 5, pp. 756-763, 2013.

[89] J. F. Hainfeld, H. M. Smilowitz, M. J. O’connor, F. A. Dilmanian, and D. N. Slatkin, "Gold nanoparticle imaging and radiotherapy of brain tumors in mice," Nanomedicine, vol. 8, no. 10, pp. 16011609, 2013.

[90] G. von Maltzahn, J. Park, A. Agrawal et al., "Computationally guided photothermal tumor therapy using long-circulating gold nanorod antennas," Cancer Research, vol. 69, no. 9, pp. 38923900, 2009.

[91] J. R. Ashton, D. P. Clark, E. J. Moding et al., "Dual-energy micro-CT functional imaging of primary lung cancer in mice using gold and iodine nanoparticle contrast agents: a validation study," PLoS ONE, vol. 9, no. 2, Article ID e88129, 2014.

[92] P. C. Naha, K. C. Lau, J. C. Hsu et al., "Gold silver alloy nanoparticles (GSAN): An imaging probe for breast cancer screening with dual-energy mammography or computed tomography," Nanoscale, vol. 8, no. 28, pp. 13740-13754, 2016.

[93] H. Wang, L. Zheng, C. Peng et al., "Computed tomography imaging of cancer cells using acetylated dendrimer-entrapped gold nanoparticles," Biomaterials, vol. 32, no. 11, pp. 2979-2988, 2011.

[94] K. Li, S. Wen, A. C. Larson et al., "Multifunctional dendrimerbased nanoparticles for in vivo MR/CT dual-modal molecular imaging of breast cancer," International Journal of Nanomedicine, vol. 8, pp. 2589-2600, 2013.

[95] C. Peng, L. Zheng, Q. Chen et al., "PEGylated dendrimerentrapped gold nanoparticles for in vivo blood pool and tumor imaging by computed tomography," Biomaterials, vol. 33, no. 4, pp. 1107-1119, 2012.

[96] H. Liu, H. Wang, Y. Xu et al., "Synthesis of PEGylated low generation dendrimer-entrapped gold nanoparticles for CT imaging applications," Nanoscale, vol. 6, no. 9, pp. 4521-4526, 2014.

[97] B. Zhou, L. Zheng, C. Peng et al., "Synthesis and characterization of PEGylated polyethylenimine-entrapped gold nanoparticles for blood pool and tumor CT imaging," ACS Applied Materials \& Interfaces, vol. 6, no. 19, pp. 17190-17199, 2014.

[98] J. Zhang, C. Li, X. Zhang et al., "In vivo tumor-targeted dual-modal fluorescence/CT imaging using a nanoprobe coloaded with an aggregation-induced emission dye and gold nanoparticles," Biomaterials, vol. 42, pp. 103-111, 2015.

[99] L. Wang, P. Zhang, J. Shi et al., "Radiofrequency-triggered tumor-targeting delivery system for theranostics application," ACS Applied Materials \& Interfaces, vol. 7, no. 10, pp. 5736-5747, 2015.

[100] J. Shi, Z. Chen, L. Wang et al., "A tumor-specific cleavable nanosystem of PEG-modified C60@Au hybrid aggregates for radio frequency-controlled release, hyperthermia, photodynamic therapy and X-ray imaging," Acta Biomaterialia, vol. 29, pp. 282-287, 2016.
[101] H. Ke, X. Yue, J. Wang et al., "Gold nanoshelled liquid perfluorocarbon nanocapsules for combined dual modal ultrasound/CT imaging and photothermal therapy of cancer," Small, vol. 10, no. 6, pp. 1220-1227, 2014.

[102] N. Chanda, R. Shukla, A. Zambre et al., "An effective strategy for the synthesis of biocompatible gold nanoparticles using cinnamon phytochemicals for phantom CT imaging and photoacoustic detection of cancerous cells," Pharmaceutical Research, vol. 28, no. 2, pp. 279-291, 2011.

[103] T. Luo, P. Huang, G. Gao et al., "Mesoporous silica-coated gold nanorods with embedded indocyanine green for dual mode $\mathrm{X}$ ray CT and NIR fluorescence imaging," Optics Express, vol. 19, no. 18, pp. 17030-17039, 2011.

[104] L. Jing, X. Liang, Z. Deng et al., "Prussian blue coated gold nanoparticles for simultaneous photoacoustic/CT bimodal imaging and photothermal ablation of cancer," Biomaterials, vol. 35, no. 22, pp. 5814-5821, 2014.

[105] F. Sousa, S. Mandal, C. Garrovo et al., "Functionalized gold nanoparticles: A detailed in vivo multimodal microscopic brain distribution study," Nanoscale, vol. 2, no. 12, pp. 2826-2834, 2010.

[106] D. P. Clark, K. Ghaghada, E. J. Moding, D. G. Kirsch, and C. T. Badea, "In vivo characterization of tumor vasculature using iodine and gold nanoparticles and dual energy micro-CT," Physics in Medicine and Biology, vol. 58, no. 6, pp. 1683-1704, 2013.

[107] B. M. Dale, A. Mark, and R. C. Semelka, MRI: Basic Principles and Applications, Wiley-Blackwell, 5th edition, 2015.

[108] M. A. Kirchin, G. P. Pirovano, and A. Spinazzi, "Gadobenate dimeglumine (Gd-BOPTA): An overview," Investigative Radiology, vol. 33, no. 11, pp. 798-809, 1998.

[109] G. Schuhmann-Giampieri, M. Mahler, G. Röll, R. Maibauer, and S. Schmitz, "Pharmacokinetics of the liver-specific contrast agent Gd-EOB-DTPA in relation to contrast-enhanced liver imaging in humans," Clinical Pharmacology and Therapeutics, vol. 37, no. 7, pp. 587-596, 1997.

[110] P. Marckmann, L. Skov, K. Rossen et al., "Nephrogenic systemic fibrosis: Suspected causative role of gadodiamide used for contrast-enhanced magnetic resonance imaging," Journal of the American Society of Nephrology, vol. 17, no. 9, pp. 2359-2362, 2006.

[111] R. Weissleder, D. D. Stark, B. L. Engelstad et al., "Superparamagnetic iron oxide: pharmacokinetics and toxicity," American Journal of Roentgenology, vol. 152, no. 1, pp. 167-173, 1989.

[112] R. Lawaczeck, H. Bauer, T. Frenzel et al., "Magnetic Iron Oxide Particles Coated with Carboxydextran for Parenteral Administration and Liver Contrasting," Acta Radiologica, vol. 38, no. 4, pp. 584-597, 2016.

[113] C. Luo, Y. Li, L. Yang, X. Wang, J. Long, and J. Liu, "Superparamagnetic iron oxide nanoparticles exacerbate the risks of reactive oxygen species-mediated external stresses," Archives of Toxicology, vol. 89, no. 3, pp. 357-369, 2015.

[114] W. Feng, X. Zhou, W. Nie et al., “Au/polypyrrole@ $\mathrm{Fe}_{3} \mathrm{O}_{4}$ nanocomposites for MR/CT dual-modal imaging guidedphotothermal therapy: An in vitro study," ACS Applied Materials \& Interfaces, vol. 7, no. 7, pp. 4354-4367, 2015.

[115] V. Amendola, S. Scaramuzza, L. Litti et al., "Magneto-plasmonic $\mathrm{Au}-\mathrm{Fe}$ alloy nanoparticles designed for multimodal SERS-MRICT imaging," Small, vol. 10, no. 12, pp. 2476-2486, 2014.

[116] D. Kim, M. K. Yu, T. S. Lee, J. J. Park, Y. Y. Jeong, and S. Jon, "Amphiphilic polymer-coated hybrid nanoparticles as CT/MRI 
dual contrast agents," Nanotechnology, vol. 22, no. 15, Article ID 155101, 2011.

[117] W. Dong, Y. Li, D. Niu et al., "A simple route to prepare monodisperse Au NP-decorated, dye-doped, superparamagnetic nanocomposites for optical, MR, and CT trimodal imaging," Small, vol. 9, no. 15, pp. 2500-2508, 2013.

[118] J. Li, L. Zheng, H. Cai et al., "Facile one-pot synthesis of Fe3O4@Au composite nanoparticles for dual-mode MR/CT imaging applications," ACS Applied Materials \& Interfaces, vol. 5, no. 20, pp. 10357-10366, 2013.

[119] L. Sun, D. Y. Joh, A. Al-Zaki et al., "Theranostic application of mixed gold and superparamagnetic iron oxide nanoparticle micelles in glioblastoma multiforme," Journal of Biomedical Nanotechnology, vol. 12, no. 2, pp. 347-356, 2016.

[120] C. Tian, L. Zhu, F. Lin, and S. G. Boyes, "Poly(acrylic acid) Bridged Gadolinium Metal-Organic Framework-Gold Nanoparticle Composites as Contrast Agents for Computed Tomography and Magnetic Resonance Bimodal Imaging," ACS Applied Materials \& Interfaces, vol. 7, no. 32, pp. 17765-17775, 2015.

[121] S. Wen, K. Li, H. Cai et al., "Multifunctional dendrimerentrapped gold nanoparticles for dual mode CT/MR imaging applications," Biomaterials, vol. 34, no. 5, pp. 1570-1580, 2013.

[122] Y. Chen, F. Guo, Y. Qiu et al., "Encapsulation of particle ensembles in graphene nanosacks as a new route to multifunctional materials," ACS Nano, vol. 7, no. 5, pp. 3744-3753, 2013.

[123] X. Shi, H. Gong, Y. Li, C. Wang, L. Cheng, and Z. Liu, "Graphene-based magnetic plasmonic nanocomposite for dual bioimaging and photothermal therapy," Biomaterials, vol. 34, no. 20, pp. 4786-4793, 2013.

[124] X. Wu, C. Li, S. Liao et al., "Silica-encapsulated Gd3+aggregated gold nanoclusters for in vitro and in vivo multimodal cancer imaging," Chemistry, vol. 20, no. 29, pp. 88768882, 2014.

[125] K. Zhang, H. Chen, P. Li et al., "Marriage strategy of structure and composition designs for intensifying ultrasound MR \& CT trimodal contrast imaging," ACS Applied Materials \& Interfaces, vol. 7, no. 33, pp. 18590-18599, 2015.

[126] Y. Liu, Z. Chang, H. Yuan, A. M. Fales, and T. VoDinh, "Quintuple-modality (SERS-MRI-CT-TPL-PTT) plasmonic nanoprobe for theranostics," Nanoscale, vol. 5, no. 24, pp. 12126-12131, 2013.

[127] A. J. Coughlin, J. S. Ananta, N. Deng, I. V. Larina, P. Decuzzi, and J. L. West, "Gadolinium-conjugated gold nanoshells for multimodal diagnostic imaging and photothermal cancer therapy," Small, vol. 10, no. 3, pp. 556-565, 2014.

[128] C. Alric, J. Taleb, G. Le Duc et al., "Gadolinium chelate coated gold nanoparticles as contrast agents for both X-ray computed tomography and magnetic resonance imaging," Journal of the American Chemical Society, vol. 130, no. 18, pp. 5908-5915, 2008.

[129] I. Lentacker, S. C. De Smedt, and N. N. Sanders, "Drug loaded microbubble design for ultrasound triggered delivery," Soft Matter, vol. 5, no. 11, pp. 2161-2170, 2009.

[130] E. G. Schutt, D. H. Klein, R. M. Mattrey, and J. G. Riess, "Injectable microbubbles as contrast agents for diagnostic ultrasound imaging: the key role of perfluorochemicals," Angewandte Chemie, vol. 42, no. 28, pp. 3218-3235, 2003.

[131] Y. Jin, J. Wang, H. Ke, S. Wang, and Z. Dai, “Graphene oxide modified PLA microcapsules containing gold nanoparticles for ultrasonic/CT bimodal imaging guided photothermal tumor therapy," Biomaterials, vol. 34, no. 20, pp. 4794-4802, 2013.
[132] D. R. Arifin, C. M. Long, A. A. Gilad et al., "Trimodal gadolinium-gold microcapsules containing pancreatic islet cells restore normoglycemia in diabetic mice and can be tracked by using US, CT, and positive-contrast MR imaging," Radiology, vol. 260, no. 3, pp. 790-798, 2011.

[133] K. Hayashi, M. Nakamura, H. Miki et al., "Gold nanoparticle cluster-plasmon-enhanced fluorescent silica core-shell nanoparticles for X-ray computed tomography-fluorescence dual-mode imaging of tumors," Chemical Communications, vol. 49, no. 46, pp. 5334-5336, 2013.

[134] D. Wu, L. Huang, M. S. Jiang, and H. Jiang, "Contrast agents for photoacoustic and thermoacoustic imaging: A review," International Journal of Molecular Sciences, vol. 15, no. 12, pp. 23616-23639, 2014.

[135] H. Li, L. Diaz, D. Lee, L. Cui, X. Liang, and Y. Cheng, "In vivo imaging of T cells loaded with gold nanoparticles: A pilot study," La Radiologia Medica, vol. 119, no. 4, pp. 269-276, 2014. 


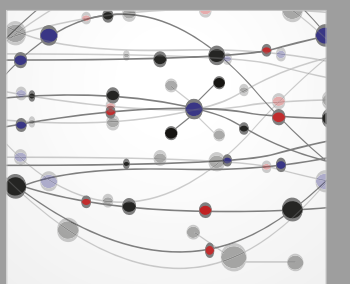

The Scientific World Journal
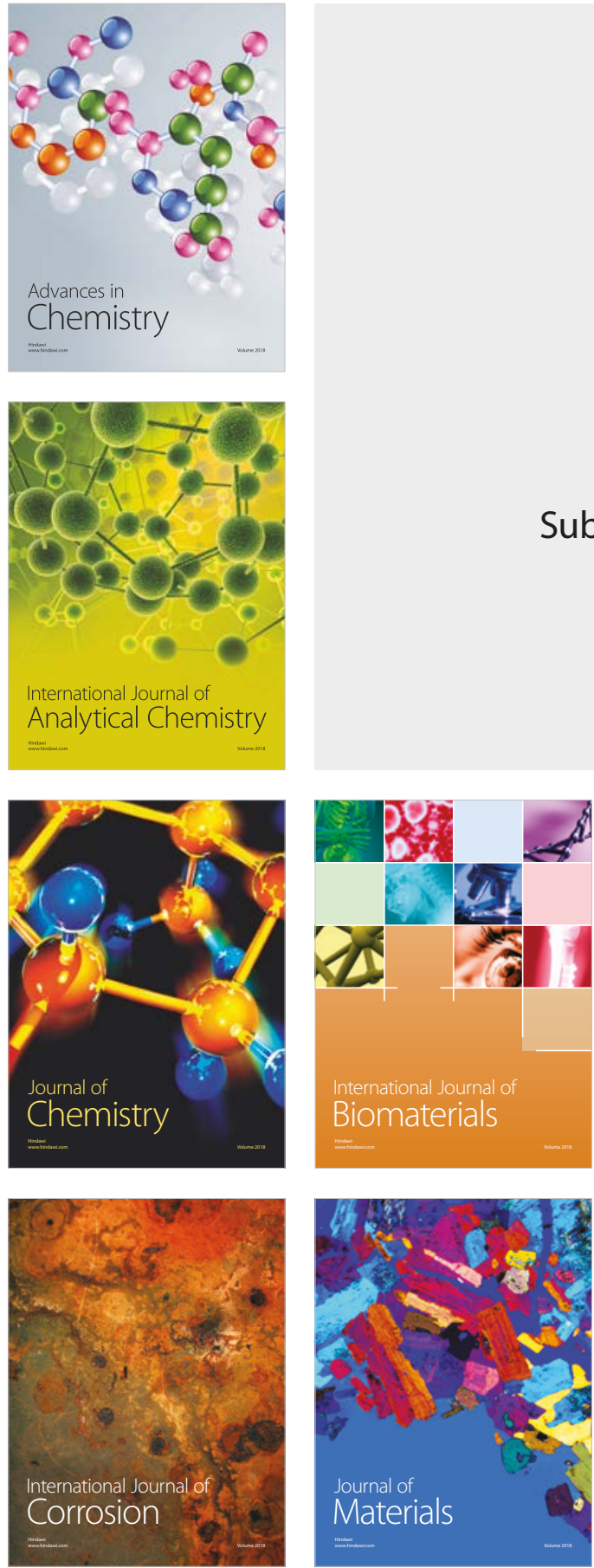

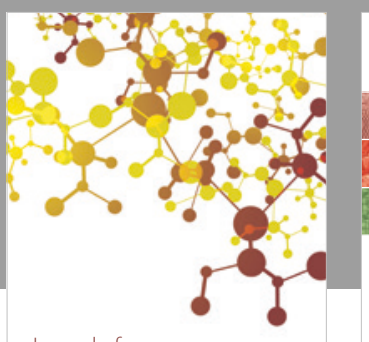

Journal of

Applied Chemistry
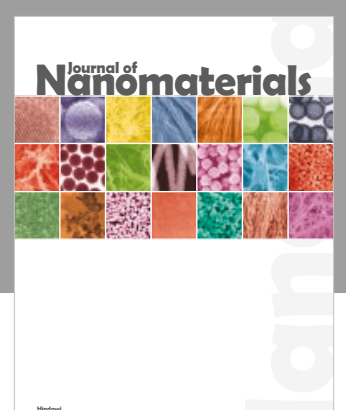

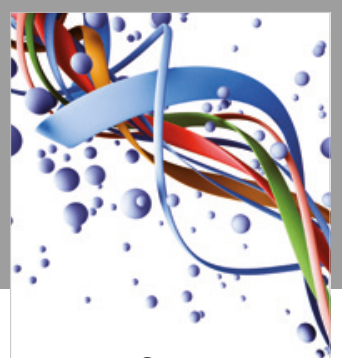

Scientifica

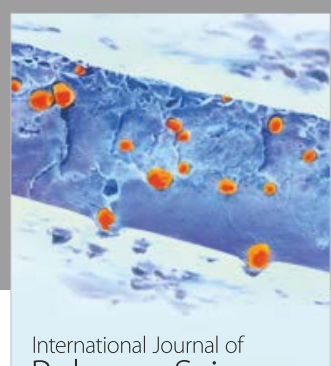

Polymer Science

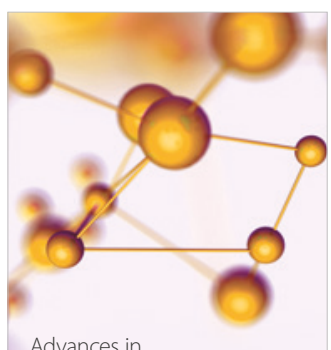

Physical Chemistry
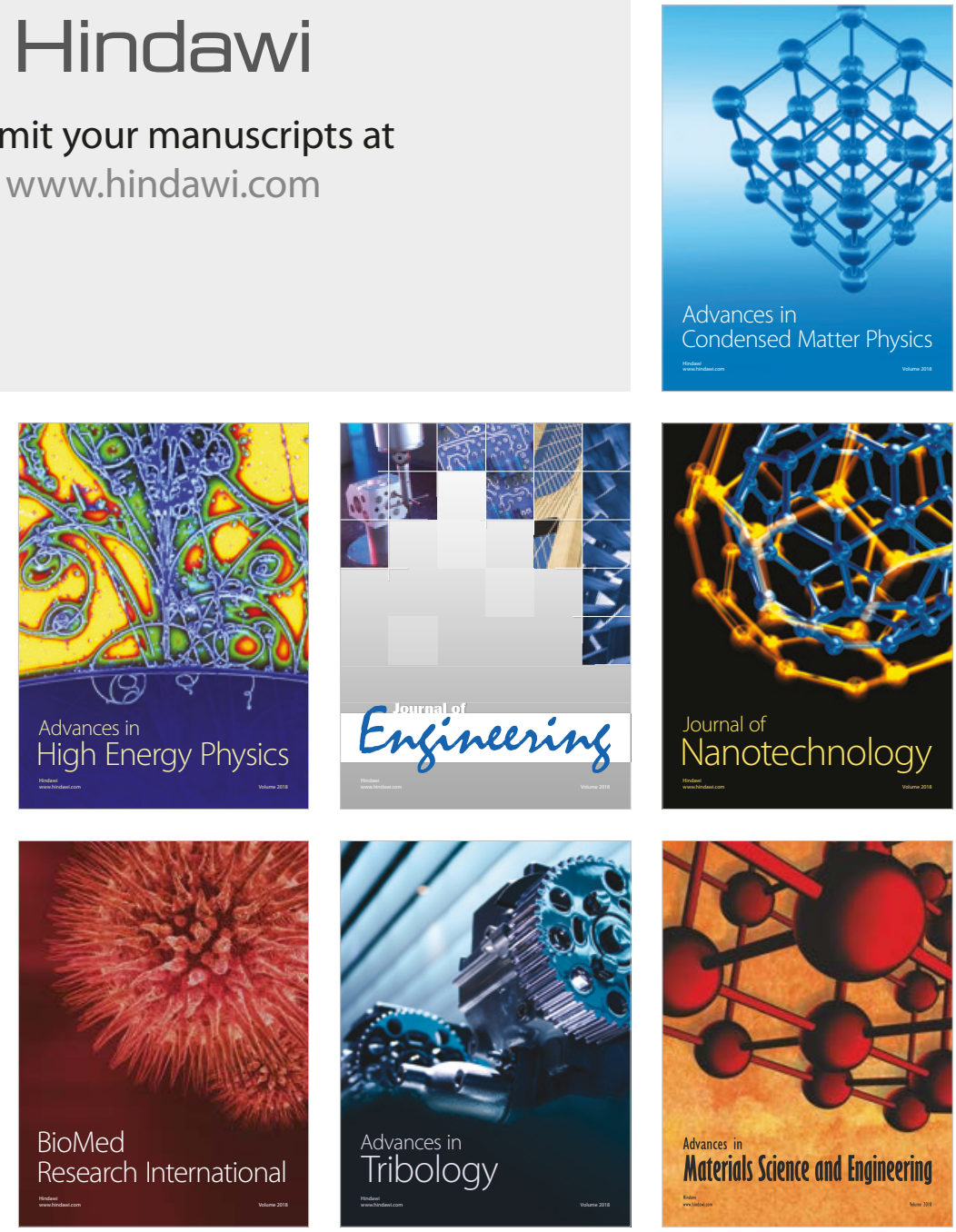